\title{
High levels of TFAM repress mammalian mitochondrial DNA transcription in vivo
}

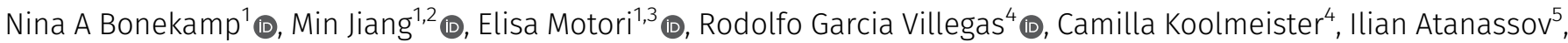 \\ Andrea Mesaros ${ }^{6}$, Chan Bae Park ${ }^{7}$, Nils-Göran Larsson ${ }^{1,4}$ (i)
}

\begin{abstract}
Mitochondrial transcription factor A (TFAM) is compacting mitochondrial DNA (dmtDNA) into nucleoids and directly controls mtDNA copy number. Here, we show that the TFAM-to-mtDNA ratio is critical for maintaining normal mtDNA expression in different mouse tissues. Moderately increased TFAM protein levels increase mtDNA copy number but a normal TFAM-to-mtDNA ratio is maintained resulting in unaltered mtDNA expression and normal whole animal metabolism. Mice ubiquitously expressing very high TFAM levels develop pathology leading to deficient oxidative phosphorylation (OXPHOS) and early postnatal lethality. The TFAM-tomtDNA ratio varies widely between tissues in these mice and is very high in skeletal muscle leading to strong repression of mtDNA expression and OXPHOS deficiency. In the heart, increased mtDNA copy number results in a near normal TFAM-to-mtDNA ratio and maintained OXPHOS capacity. In liver, induction of LONP1 protease and mitochondrial RNA polymerase expression counteracts the silencing effect of high TFAM levels. TFAM thus acts as a general repressor of mtDNA expression and this effect can be counterbalanced by tissue-specific expression of regulatory factors.
\end{abstract}

DOI 10.26508/lsa.202101034 | Received 25 January 2021 | Revised 10 August 2021 | Accepted 20 August 2021 | Published online 30 August 2021

\section{Introduction}

Treatment of mitochondrial diseases caused by dysfunctional oxidative phosphorylation (OXPHOS) remains a challenge. The clinical variability is substantial as almost any organ and cell type may be affected with varying degrees of severity; with disease onset varying from the neonatal period to late in adult life. Moreover, mutations in two different genomes, that is, nuclear DNA and mitochondrial DNA (mtDNA), can cause OXPHOS defects and thus mitochondrial disease (Rahman, 2020; Russell et al, 2020). The majority of mitochondrial proteins ( 99\%), including all that regulate mtDNA maintenance and expression, are encoded in the nucleus and imported into mitochondria. In contrast, mtDNA only contributes 13 proteins that all have essential roles for the function of four of the OXPHOS complexes. Mammalian mtDNA is a compact circular double-stranded genome of about $16.6 \mathrm{~kb}$ in size, where each strand undergoes polycistronic transcription followed by RNA processing and maturation to yield 2 ribosomal RNAs (rRNAs), 22 tRNAs, and 11 mRNAs (translated to 13 proteins) (Anderson et al, 1981; Bibb et al, 1981; Gustafsson et al, 2016). In the last $30 \mathrm{yr}$, substantial progress has been made in understanding the genetic basis of mitochondrial diseases. More than 300 different pathogenic point mutations, deletions and duplications of mtDNA have been identified since the first disease-causing mtDNA mutations were reported (Holt et al, 1988; Wallace et al, 1988). Because of the high copy number of mtDNA, pathogenic mutations may affect all (homoplasmy) or only a subset (heteroplasmy) of all mtDNA molecules in a cell (Sciacco et al, 1994; Taylor \& Turnbull, 2005). A biochemical phenotype is induced once a certain threshold level of mutant mtDNA is exceeded. To add to the complexity, mutations in about 300 nuclear genes have been shown to cause mitochondrial disorders by affecting different aspects of mitochondrial function, such as biogenesis of the OXPHOS system, nucleotide transport/synthesis, membrane dynamics, mtDNA maintenance, and mtDNA expression at different levels, including transcription, RNA maturation, and translation (Vafai \& Mootha, 2012; Thompson et al, 2020).

The use of animal models has facilitated our understanding of mitochondrial diseases and given insights into a variety of potential treatment options (Russell et al, 2020), for example, important pathophysiological features of mitochondrial myopathy were recapitulated in mice with a disruption in mtDNA expression in skeletal muscle (Wredenberg et al, 2002). Importantly, a substantial increase in mitochondrial mass was demonstrated to partly

\footnotetext{
${ }^{1}$ Department of Mitochondrial Biology, Max Planck Institute for Biology of Ageing, Cologne, Germany ${ }^{2}$ Zhejiang Provincial Laboratory of Life Sciences and Biomedicine, Key Laboratory of Growth Regulation and Transformation Research of Zhejiang Province, School of Life Sciences, Westlake University, Hangzhou, China ${ }^{3}$ Cologne Excellence Cluster on Cellular Stress Responses in Aging-Associated Diseases (CECAD), Cologne, Germany ${ }^{4}$ Department of Medical Biochemistry and Biophysics, Karolinska Institutet, Stockholm, Sweden ${ }^{5}$ Proteomics Core Facility, Max Planck Institute for Biology of Ageing, Cologne, Germany ${ }^{6}$ Phenotyping Core Facility, Max Planck Institute for Biology of Ageing, Cologne, Germany ${ }^{7}$ Ajou University, Suwon, Republic of Korea
}

Correspondence: nina.bonekamp@medma.uni-heidelberg.de; nils-goran.larsson@ki.se Nina A Bonekamp's present address is Department of Neuroanatomy, Mannheim Center for Translational Neuroscience (MCTN), Medical Faculty Mannheim, Heidelberg University, Mannheim, Germany 
compensate for reduced OXPHOS function by maintaining the overall ATP production at near-normal levels despite poor function of individual mitochondria in skeletal muscle (Wredenberg et al, 2002). Furthermore, boosting mitochondrial biogenesis has become a promising approach exploited for the treatment of mitochondrial disease (Whitaker et al, 2016). Overexpression of PPAR-y coactivator $1-\alpha$ (PGC1 $\alpha$ ), an important regulator of mitochondrial biogenesis (Puigserver et al, 1998; Wu et al, 1999; Scarpulla, 2002), can improve mitochondrial disease manifestations in mice (Viscomi et al, 2011; Dillon et al, 2012). Because of the complex regulation of PGC1 $\alpha$ activity (Fernandez-Marcos \& Auwerx, 2011), direct targeting of downstream effectors of the PGC1 $\alpha$ signalling cascade, such as the mitochondrial transcription factor A (TFAM) (Virbasius \& Scarpulla, 1994), might provide a more directed treatment approach.

TFAM is an essential regulator of mitochondrial function in mammals because of its dual role as a core component of the mitochondrial transcription initiation machinery and as the key factor packaging mtDNA into mitochondrial nucleoids (Falkenberg et al, 2002; Alam et al, 2003; Kukat et al, 2011, 2015; Shi et al, 2012). The TFAM protein consists of two high mobility group-box domains that are separated by a linker and followed by a short C-terminal tail essential for transcription activation (Fisher \& Clayton, 1988; Parisi \& Clayton, 1991; Dairaghi et al, 1995; Kanki et al, 2004). Each of the two high mobility group box domains causes mtDNA to bend $90^{\circ}$ leading to a $180^{\circ} \mathrm{U}$-turn when one molecule of TFAM binds mtDNA (Ngo et al, 2011; Rubio-Cosials et al, 2011). Specific binding of TFAM to the two mtDNA promoters results in transcription activation by recruitment of the mitochondrial RNA polymerase (POLRMT) and mitochondrial transcription factor B2 (TFB2M) (Hillen et al, 2017). Nonsequence-specific TFAM binding enables packaging of the mitochondrial genome into protein-DNA complexes to form the mitochondrial nucleoid (Shen \& Bogenhagen, 2001; Alam et al, 2003; Kukat et al, 2011, 2015; Bonekamp \& Larsson, 2018). Importantly, TFAM levels are known to directly control mtDNA copy number (Larsson et al, 1998; Matsushima et al, 2003; Ekstrand et al, 2004; Kanki et al, 2004) and multiple in vivo studies have shown that TFAM overexpression is beneficial in mouse models with various types of pathology, for example, myocardial infarction, amyotrophic lateral sclerosis, transient forebrain ischemia and age-dependent memory loss (Ikeuchi et al, 2005; Hayashi et al, 2008; Hokari et al, 2010; Morimoto et al, 2012). Increasing the absolute levels of mtDNA has also been shown to improve the function in certain organs in mouse models of mitochondrial disease and premature ageing (Nishiyama et al, 2010; Jiang et al, 2017; Filograna et al, 2019, 2021). Remarkably, the rescue effect has been shown to occur although the proportion of mutant mtDNA stays the same (Jiang et al, 2017; Filograna et al, 2019), thus showing that the absolute levels of wild-type mtDNA play an important role in determining pathogenic effects caused by heteroplasmic mtDNA mutations.

Based on the findings in mouse models, it has been suggested that manipulation of TFAM levels and thus mtDNA copy number is a target for disease intervention. However, increased mtDNA copy number has also been reported to result in enlarged nucleoids, inhibition of mitochondrial transcription and respiratory chain dysfunction (Ylikallio et al, 2010). There are a number of limitations in the abovementioned studies that do not allow a definite conclusion on whether up-regulation of mtDNA copy number by TFAM overexpression is beneficial or detrimental in vivo. Several studies have relied on a transgenic mouse model expressing the human TFAM CDNA from the synthetic CAG (modified chicken $\beta$-actin promoter with CMV-IE enhancer) promoter that causes robust expression of the human TFAM protein in the heart, skeletal muscle, and brain, but barely detectable expression in lung, liver and kidney (Ikeuchi et al, 2005; Hayashi et al, 2008; Hokari et al, 2010; Ylikallio et al, 2010; Morimoto et al, 2012). This tissue-specific expression pattern is likely explained by a positional effect caused by the random genomic integration of the human TFAM cDNA construct. Another complication of this experimental system is that it is heterologous as the human TFAM protein, which only poorly activates mouse mtDNA transcription (Ekstrand et al, 2004), is expressed in the mouse. As a consequence, the mutant mice display an increase in mtDNA levels without increasing OXPHOS or mitochondrial mass (Ekstrand et al, 2004). In contrast, TFAM can stimulate mtDNA expression in an autologous system as it has been reported that import of human TFAM into human mitochondria stimulates mtDNA transcription (Garstka et al, 2003; Maniura-Weber et al, 2004).

Here, we describe a series of mouse models with a moderate or strong overexpression of the endogenous mouse TFAM protein and investigate the effects on mtDNA copy number, mitochondrial gene expression and whole animal physiology. We show that moderate increase in TFAM is well tolerated in the mouse, whereas strong overexpression has deleterious consequences in certain tissues. The most severe phenotype was observed in tissues where the mtDNA copy number remained low, which resulted in a high TFAM to mtDNA ratio that completely abolished mitochondrial gene expression. Thus, although TFAM is essential for transcription initiation, it also acts a general gene repressor of mtDNA expression in mammalian mitochondria in vivo. Modulation of TFAM levels thus serves as a global mechanism to regulate mitochondrial gene expression likely by influencing nucleoid compaction.

\section{Results}

\section{Moderately increased TFAM levels sustain normal mtDNA expression}

To study the consequences of moderately increased TFAM levels in vivo, we generated bacterial artificial chromosome (BAC) transgenic mice harbouring an introduced $203 \mathrm{~kb}$ mouse genomic fragment containing Tfam expressed from its endogenous promoter under the control of adjacent regulatory elements (Fig 1A). Three lines of BAC-TFAM transgenic mice derived from independent founders were maintained as heterozygotes on an inbred C57Bl/6N background. BAC constructs are typically randomly inserted into the mouse genome and analysis of the three different founder lines, BAC TG 137 (Figs 1 and S1), BAC TG 188 (Fig S1), and BAC TG 91 (Fig S1) allowed us to rule out effects caused by insertional mutagenesis. Western blot analyses of total tissue extracts revealed a moderate overexpression of TFAM of 1.63-fold in the heart, 1.50-fold in the liver, and 1.49-fold in the skeletal muscle in the BAC TG 137 line compared to control (Fig 1B). The other founder lines also displayed an increase in TFAM protein levels in all tissues 
A

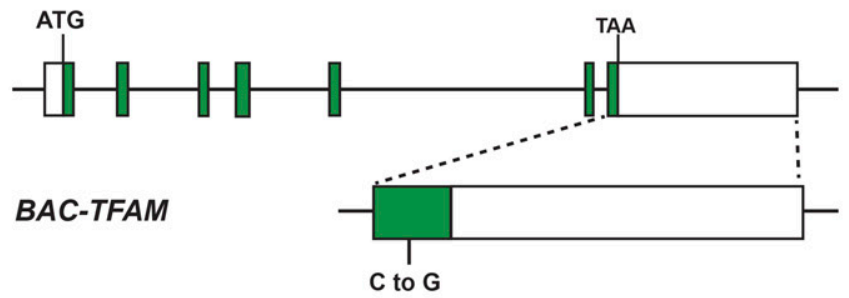

C

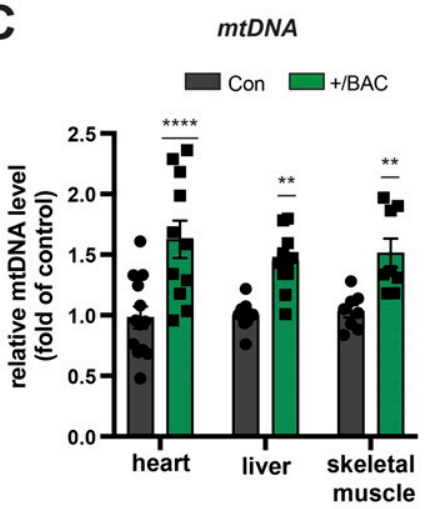

D

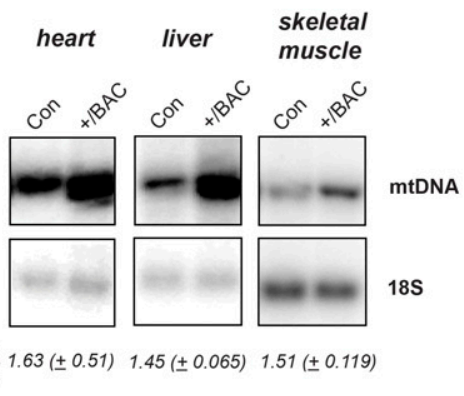

$m t D N A$
levels:

B

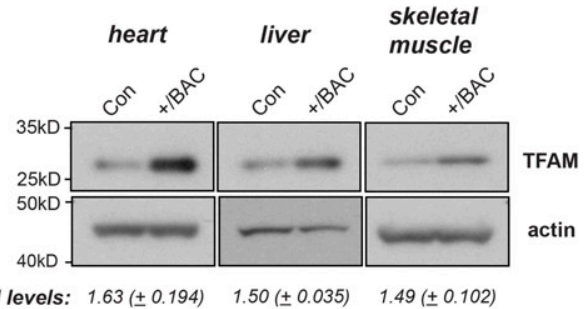

$\mathbf{E}$

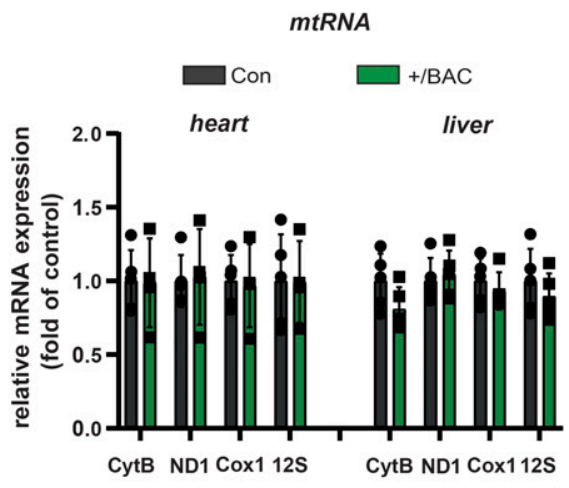

H

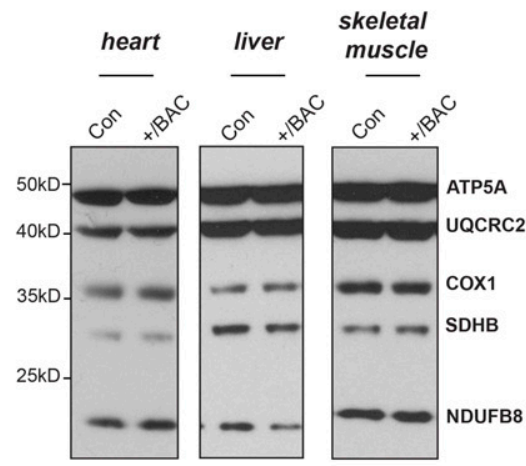

52 weeks

G

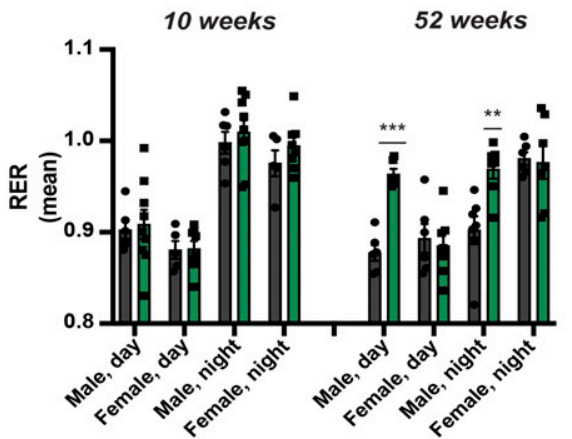

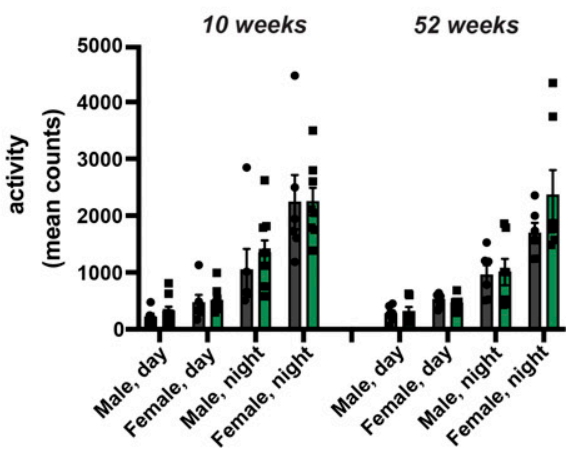

Figure 1. Moderate increase in TFAM levels leads to increased mtDNA copy number without effects on mitochondrial gene expression.

(A) Overview of the bacterial artificial chromosome (BAC) construct expressing mouse Tfam under its endogenous promoter. Green boxes indicate Tfam exons. A neutral point mutation generating a Pvul restriction site was introduced to distinguish the BAC from the Tfam wild-type locus (indicated as $C$ to $G$ in the magnified exon). (B) Western blot analysis of TFAM protein levels in the heart, liver, and skeletal muscle whole cell lysates of wild-type (Con) and BAC-TFAM TG 137 (+/BAC) animals. The BAC TG 137 founder line is used in all subsequent experiments and referred to as +/BAC. Actin was used as a loading control. A representative image is shown ( $n=3$ independent experiments). (C) Quantification of steady-state mtDNA levels in the heart, liver, and skeletal muscle of wild-type (Con) and BAC-TFAM (+/BAC) animals. In the case of heart and liver, mtDNA levels were quantified by qPCR using specific probes against COX1 and 18S. Quantification of skeletal muscle mtDNA levels was performed by densitometric analysis of Southern blots. Data are expressed as means \pm SEM $(n=9-12$ biological replicates for heart and liver; $n=8$ for skeletal muscle; $P<0.01:{ }^{* *} ; P<0.0001:{ }^{* * * *}$, two-way ANOVA with Sidak's test for multiple comparisons). (D) Southern blot analysis of Pstl-digested mtDNA derived from the heart, liver, and skeletal muscle of wild-type (Con) and BAC-TFAM (+/BAC) animals. mtDNA was quantified by radiolabeling with a specific probe against COX1, nuclear DNA was probed with 18S. A representative image is shown ( $n=3$ independent experiments). (E) Analysis of steady-state mitochondrial transcript levels in heart and liver of wild-type (Con) and BAC-TFAM (+/BAC) animals by qRT-PCR. Mitochondrial mRNAs and rRNAs were quantified using specific mouse probes, $\beta$-2-microglobulin was used as a reference gene. ( $n=4-5$ biological replicates). (F) Western blot analysis of steady-state levels of respiratory chain subunits in the heart, liver, and skeletal muscle mitochondrial extracts of wild-type (Con) and BAC-TFAM (+/BAC) animals. A representative image is shown ( $\mathrm{n}=3$ independent experiments). (G, $\mathbf{H})$ Phenotyping/energy homeostasis of BAC-TFAM mice aged 10 and $52 \mathrm{wk}$. Cohorts of BAC-TFAM mice (+/BAC) and wild-type litter mates (Con) were analysed by indirect calorimetry at the age of 10 and $52 \mathrm{wk}$. Data on respiratory exchange rate (RER, [G]) and activity (sum of ambulatory and fine movement, $[\mathrm{H}]$ ) are shown (means $\pm \mathrm{SEM}, \mathrm{n}=5-8$ biological replicates; $P<0.01$ : **; $P<0.001$ : ***, Two-way ANOVA with Sidak's test for multiple comparisons).

Source data are available for this figure.

investigated, albeit at different levels. In BAC TG 188, a similar level of TFAM expression was observed in heart (1.78-fold), with lower levels in liver and skeletal muscle (Fig S1A and B). In BAC TG 91, we observed a consistent increase in TFAM levels in all tissues, the highest levels being detected in skeletal muscle (1.68-fold). We next investigated the relative mtDNA copy number in different tissues using quantitative PCR (qPCR) and Southern blotting. In line with the well-established role of TFAM as a key regulator of mtDNA copy 
number, increased TFAM levels led to a significant increase in mtDNA up to 1.63-fold of control in the heart, 1.45-fold in the liver, and 1.51-fold in the skeletal muscle in BAC TG 137 mice (Fig 1C and D). The relative mtDNA copy number was similarly increased in the other founder lines and depended on the TFAM levels (Fig S1C and D). Because of the consistent, moderate TFAM increase observed in the different tissues of the heterozygous BAC TG 137 line (Jiang et al, 2017; Filograna et al, 2019), we proceeded to extensively characterize this line, henceforth denoted BAC-TFAM (+/BAC) mice.

Normally, TFAM is present in around 1,000 molecules per mtDNA molecule or 1 TFAM molecule per 16-17 bp of mtDNA in mammalian cells (Kukat et al, 2011). High TFAM-to-mtDNA ratios have been shown to block mitochondrial gene expression in vitro (Farge et al, 2014). In the different tissues of BAC-TFAM mice, the TFAM-to-mtDNA ratio was maintained at the same relative levels as in wild-type mice (1.00 in the heart, 1.03 in the liver, and 0.98 in the skeletal muscle), which should not affect mitochondrial gene expression.

We addressed the steady-state mtDNA transcript levels by quantitative reverse transcription PCR ( $R$ RT-PCR) and Northern blotting (Figs $1 \mathrm{E}$ and S1E and F). The mitochondrial mRNA, rRNA and tRNA levels were not changed in heart or liver tissue of BAC-TFAM mice (Figs $1 \mathrm{E}$ and S1E and F) or in the BAC TG 188 or BAC TG 91 mouse lines (Fig S1E and F), showing that a moderate increase in TFAM expression does not globally affect mtDNA transcription. In line with this, Western blotting of isolated mitochondria from the heart, liver, and skeletal muscle of BAC-TFAM mice showed no change in the expression of OXPHOS subunits (Fig 1F). Furthermore, tandem mass tag (TMT)-based quantitative proteomics on whole tissue lysates of the heart, liver, and spleen of BAC-TFAM mice confirmed that a moderate increase in TFAM protein levels does not affect the total cellular proteome and is therefore unlikely to affect normal tissue function (Fig S2A). Thus, moderate TFAM overexpression causes an increase in mtDNA copy number without affecting overall mtDNA gene expression.

\section{Moderately increased TFAM and mtDNA levels are well tolerated in vivo}

We proceeded to assess physiology in the BAC-TFAM mice at different ages. To this end, we measured energy homeostasis and activity of BAC-TFAM animals and wild-type litter mates at the age of 10 and 52 wk using metabolic cages. This enabled us to investigate their metabolic performance, spontaneous locomotor activities and drinking and feeding behaviour. We did not observe substantial changes in the drinking and feeding behaviour or body weight between wild-type and BAC-TFAM mice of both sexes at 10 and 52 wk of age (Fig S2B-D). The normal weight and food intake in BAC-TFAM mice indicated that they do not suffer from stress, disease conditions or metabolic changes. The measurements of $\mathrm{O}_{2}$ consumption and $\mathrm{CO}_{2}$ production in metabolic cages were used to calculate the respiratory exchange rate (Fig 1G) and the energy expenditure (heat; Fig S2E) that both were within a normal range in BAC-TFAM mice. We observed a significant increase in the respiratory exchange rate in BAC-TFAM males aged $52 \mathrm{wk}$, which points towards a preferred utilization of carbohydrates in those mice (Fig 1G). This increase did not correlate with a change in food intake or increased activity, as animals did not display any substantial alteration in activity, given as the sum of ambulatory and fine movements (Fig $1 \mathrm{H})$ and the cumulative distance travelled (Fig S2F). The underlying physiological difference and sex-specificity requires further investigation. Litter sizes were within the normal range of C57Bl6N mice for the BAC-TFAM, BAC TG 188, and BAC TG 91 mouse lines (Fig S2G), showing that the reproductive performance and fertility was not impaired. We thus conclude that BAC-TFAM mice are healthy and indistinguishable from controls and that a moderate increase in TFAM protein levels and mtDNA copy number is very well tolerated in vivo without affecting animal physiology or metabolism.

\section{Strong TFAM overexpression results in postnatal lethality}

Next, we investigated the effects of strong TFAM overexpression on mtDNA copy number and mitochondrial function in vivo. To this end, we generated knock-in mice that can activate the expression of a FLAG-tagged mouse TFAM protein under the control of the CAG promoter in the ROSA26 locus. The ROSA26 locus is known to provide ubiquitous expression and the CAG promoter is a commonly used synthetic promoter driving high levels of transgene expression in almost all cell types of transgenic animals (Niwa et al, 1991; Okabe et al, 1997). Mice heterozygous for the CAG-TFAM allele preceded by a loxp-flanked stop-cassette were mated to mice ubiquitously expressing cre-recombinase ( $\beta$-actin-cre) to generate mice overexpressing mouse TFAM in all tissues, henceforth denoted CAG-TFAM mice (Fig 2A). The litter sizes of CAG-TFAM mice were normal and the mutant mice were born at the expected Mendelian ratios (Fig S3A). To our surprise, we observed increased lethality of CAG-TFAM mice after postnatal day 16 and all mice died or had to be euthanized before postnatal day 35 (Fig 2B). CAG-TFAM mice were generally smaller and weaker than wild-type litter mates (Fig 2C) and the heart, liver, and kidney were smaller in comparison with wild-type animals (Fig S3B and C). Strong TFAM overexpression thus had a profound effect on animal well-being and we therefore investigated the underlying cause of this detrimental effect on a tissue and cellular level.

Combined cytochrome c oxidase/succinate dehydrogenase enzyme activity (COX/SDH) staining in heart and skeletal muscle of CAG-TFAM mice revealed a pronounced loss of COX-reactive skeletal muscle fibres and a smaller number of COX-negative cardiomyocytes in the heart (Fig 2D). This finding points to a severe mitochondrial dysfunction in muscle tissue. To address a direct link between high TFAM protein levels and the resulting mitochondrial dysfunction, we first ruled out an indirect effect caused by a possible oversaturation of the mitochondrial protein import system. Cytosolic accumulation of mitochondrial precursors has been shown to be hazardous to cellular fitness and can trigger an adaptive response to counteract this cytosolic protein stress (Boos et al, 2019). We reasoned that saturation of the mitochondrial import system, if present, would lead to an increase in mitochondrial precursor proteins in the cytosol and a global decrease in matrix proteins. We detected a similar pattern of aconitase (ACO2) distribution between the cytosol and mitochondria after subcellular fractionation of heart and liver tissue in control and CAG-TFAM animals (Fig S3D). Furthermore, Western blot analysis of mitochondrial proteins with a well-defined and prominent cleavable mitochondrial targeting sequence showed no increase in the levels of non-imported precursors in addition to the mature proteins in the heart, liver, and skeletal muscle tissue extracts, even after 
A

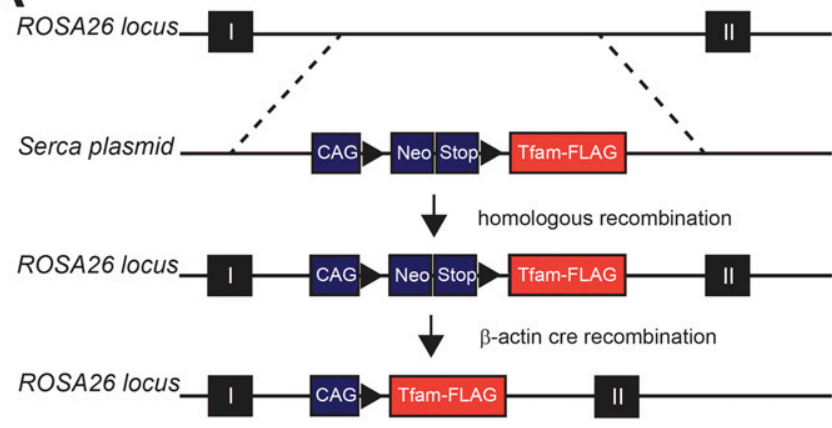

CAG-TFAM
B

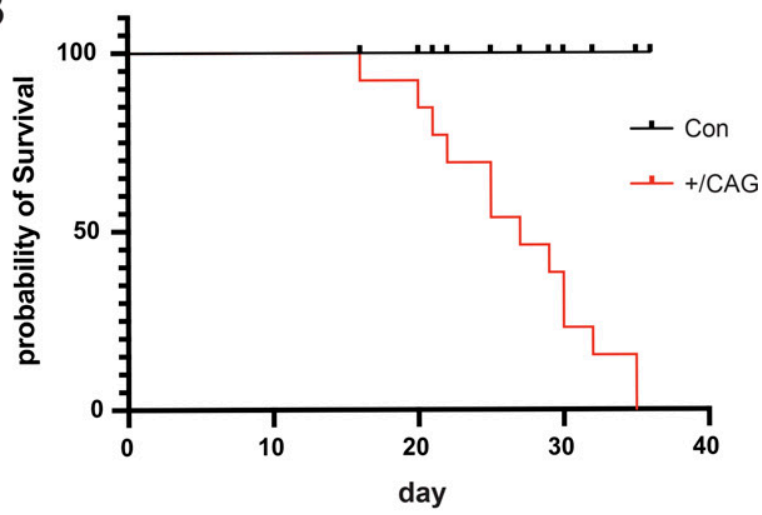

C

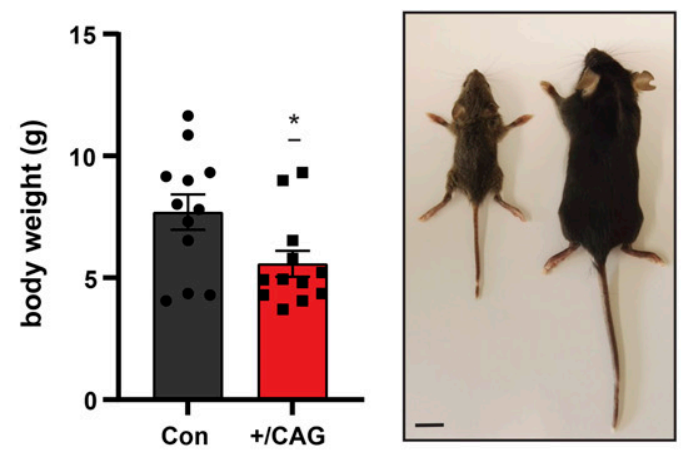

D

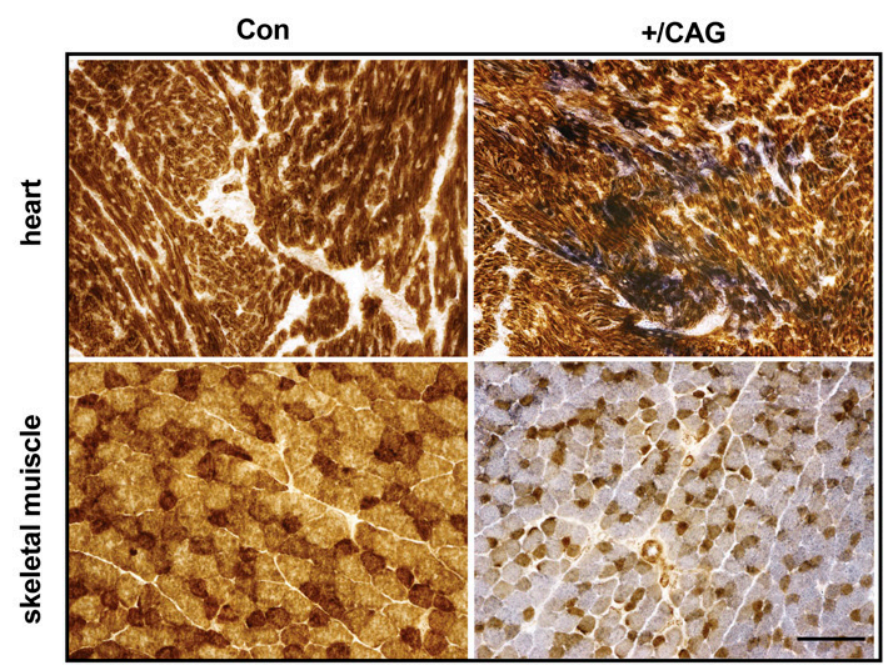

Figure 2. High TFAM overexpression leads to early postnatal mortality.

(A) Strategy to generate CAG-TFAM mice. A CDNA construct encoding a FLAG-tagged TFAM protein under the control of the CAG promoter was introduced into the ROSA26 locus by homologous recombination. CAG-TFAM mice were generated by crossing to $\beta$-actin cre animals. (B) Survival curve of CAG-TFAM mice. Litters yielding CAG-TFAM mice (+/CAG) were observed for $40 \mathrm{~d}$ for development and survival compared with control litter mates (Con) $(n=12)$. (C) Body weight of CAG-TFAM mice $(+/$ CAG) compared with control litter mates (Con) at the age of 3 wk. Means $\pm S E M, n=12$ biological replicates; $P<0.05: *$, unpaired $t$ test. Scale bar, $1 \mathrm{~cm}$. (D) COX/SDH staining of heart (upper panel) and skeletal muscle (lower panel) of CAG-TFAM mice $(+/$ CAG) compared with control litter mates (Con) at the age of 3 wk. Representative images are shown ( $n=3$ biological replicates). Scale bar, $100 \mu \mathrm{m}$.

Source data are available for this figure.

prolonged exposure of blots (Fig S3E). In addition, the prominent precursor sequences of ATP5A and NDUFA9 were not detectable by quantitative TMT proteomics of whole tissue extracts from the heart, liver, and skeletal muscle (Fig S3F). Our quantitative proteomics data also showed no general defects in mitochondrial protein levels, but rather mildly increased levels of proteins involved in the tricarboxylic acid (TCA) cycle, lipid, and acetyl CoA metabolism, iron-sulphur cluster synthesis, and heme synthesis (Fig S3G). We thus conclude that strong TFAM expression exceeding the normal physiological range can directly shut down mitochondrial function without affecting mitochondrial protein import.

\section{High TFAM-to-mtDNA ratios abolish mtDNA expression in skeletal muscle}

We proceeded to analyse the underlying molecular basis for the observed mitochondrial dysfunction in different tissues from CAG-
TFAM mice. Mitochondrial metabolism and function is different between tissues, and mitochondria from different organs therefore differ in their biosynthetic capacity and ultrastructural appearance (Vafai \& Mootha, 2012). Western blotting of total tissue extracts from CAG-TFAM mice revealed that the relative TFAM expression was increased 4.46-fold in heart and 3.84-fold in skeletal muscle in comparison with wild-type mice (Fig $3 A$ and $B$ ). These levels much exceeded the TFAM levels observed in BAC-TFAM mice (Fig 1B). Although the relative TFAM overexpression levels were comparable in both heart and skeletal muscle in CAG-TFAM mice, the mitochondrial dysfunction was much more severe in skeletal muscle than in heart (Fig 2D). Changes in the TFAM-to-mtDNA ratio affect mitochondrial function in vitro, so we proceeded to analyse potential differences in mtDNA levels between the two tissues by qPCR and Southern blotting. We observed markedly increased mtDNA levels in heart (6.35-fold of control), whereas the mtDNA copy number (0.96-fold of control) was not changed in skeletal muscle 

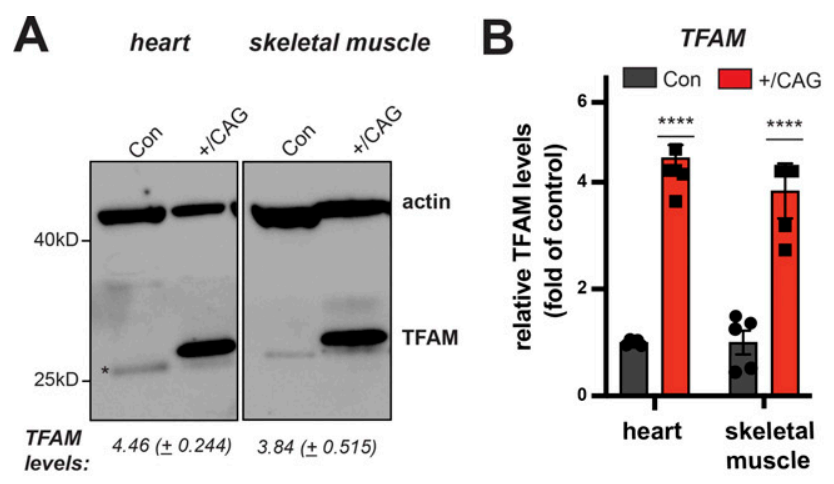

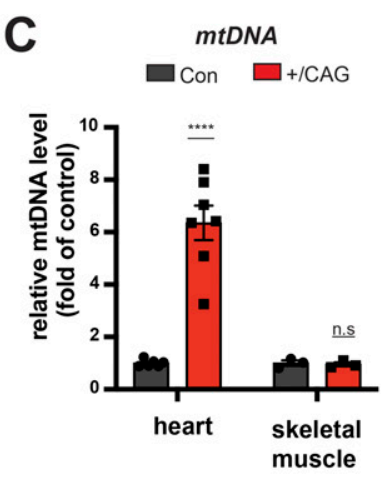

D heart skeletal muscle

E

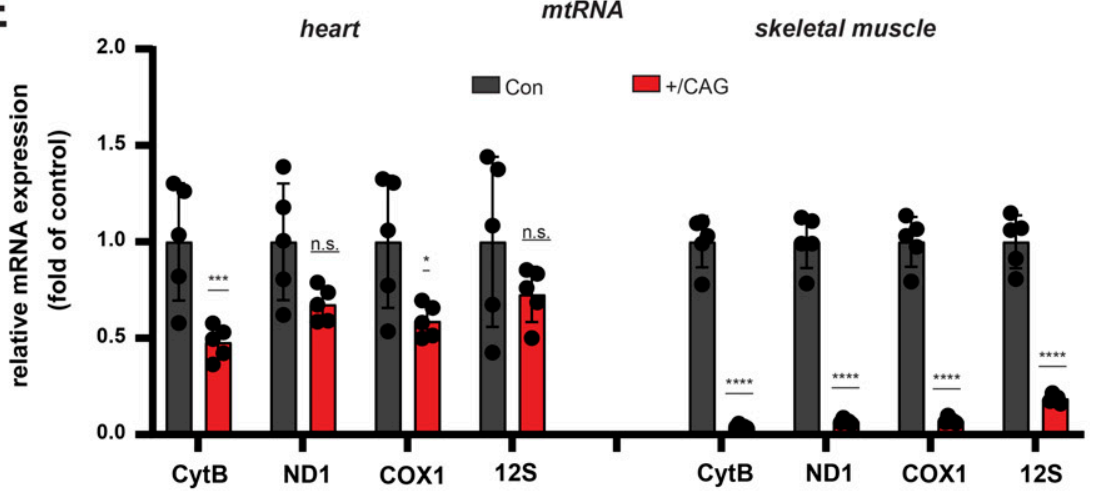

$\mathbf{F}$
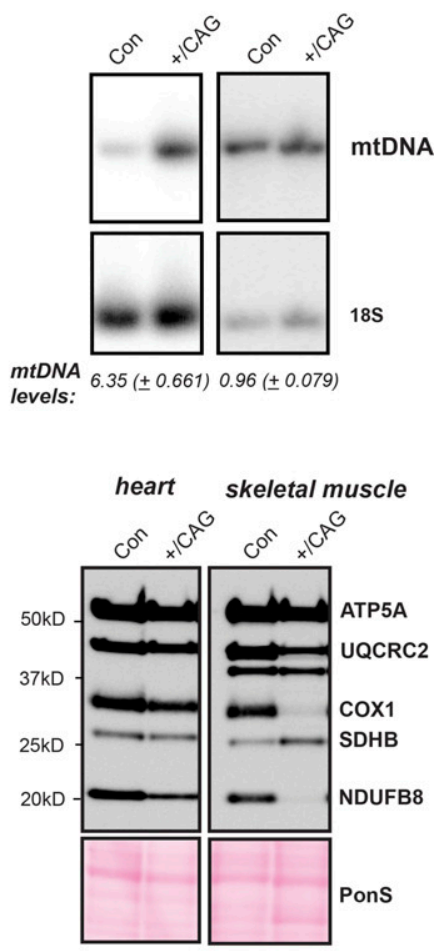

G

heart

skeletal muscle
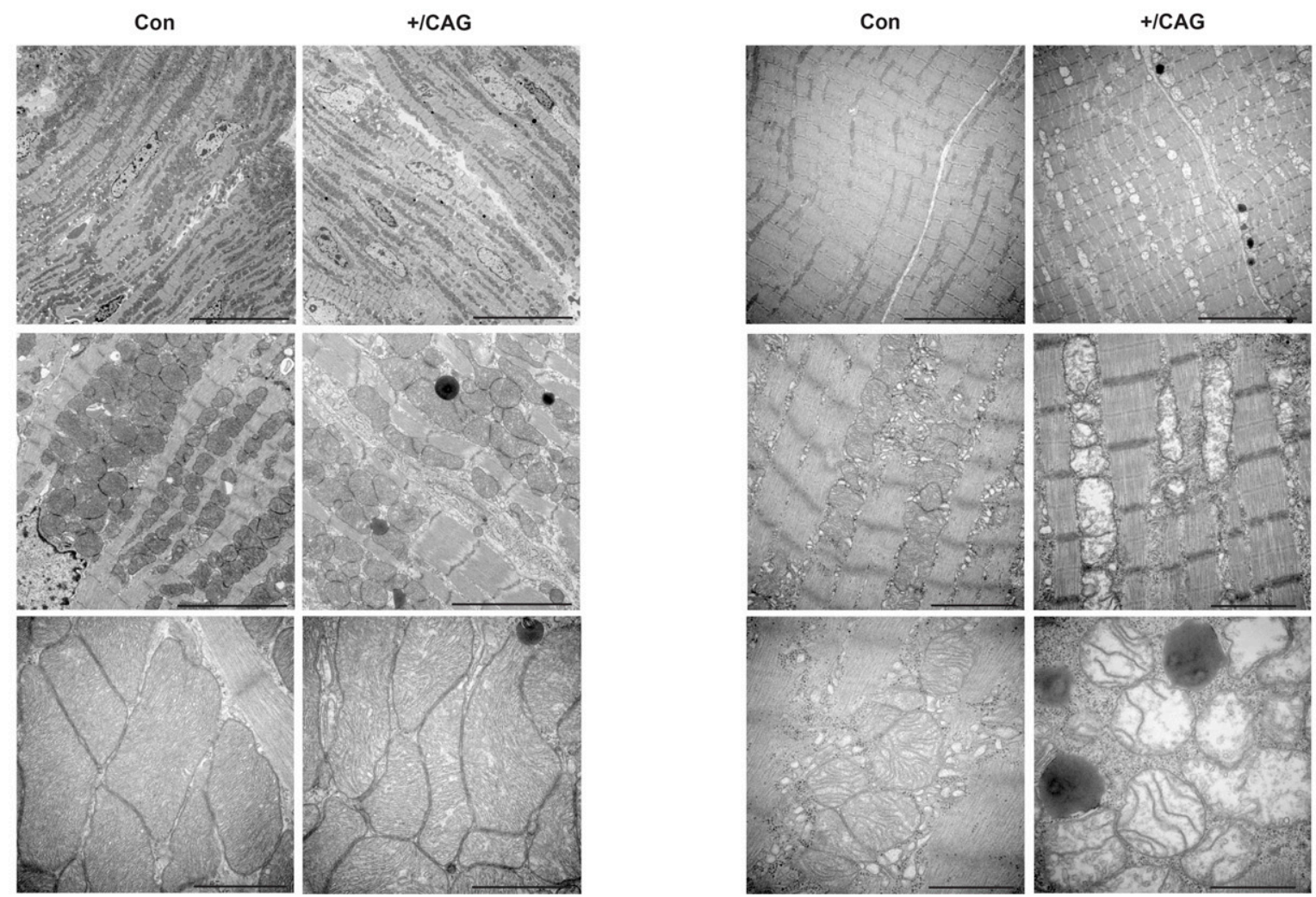
despite the strong increase in TFAM protein levels (Fig $3 C$ and D). The relative TFAM-to-mtDNA ratio was therefore markedly increased to 3.96-fold (3.84 TFAM:0.96 mtDNA) in skeletal muscle. These findings indicate that nucleoids in skeletal muscle of CAGTFAM mice may be too saturated with TFAM and too compacted to allow sufficient transcription for normal mtDNA expression. qRT-PCR of mitochondrial steady-state transcript levels indeed confirmed a severe depletion of mitochondrial mRNAs and rRNAs in skeletal muscle of CAG-TFAM mice (Fig 3E). We also observed a moderate decrease in CytB and Cox1 transcript levels in the heart, despite the strong mtDNA copy number increase (Fig 3D). In line with this, OXPHOS protein levels were only slightly decreased in heart mitochondria, whereas Western blotting of OXPHOS protein subunits in isolated skeletal muscle mitochondria revealed a strong depletion of the mitochondrially encoded COX1 subunit of Complex IV and the nucleus-encoded NDUFB8 subunit of Complex I. The levels of the UQCRC2 subunit of Complex III were mildly decreased (Fig 3F).

To determine the effects of altered TFAM-to-mtDNA ratios on mitochondrial and tissue organization, we performed transmission electron microscopy of tissue sections from heart and skeletal muscle (Fig 3G). In heart, we observed an altered mitochondrial morphology with less densely packed cristae, however, the overall organization and structure of heart tissue remained unchanged (Fig 3G, left). Cristae arrangement and nucleoid distribution were recently shown to be intrinsically linked by a highly ordered coorganization (Stephan et al, 2019). Thus, we investigated mitochondrial nucleoids in heart sections by stimulated emission depletion (STED) microscopy in CAG-TFAM mice. The nucleoid numbers in CAG-TFAM mice were markedly increased in line with the observed increase in mtDNA copy number, but the nucleoids formed extensive clusters (Fig S4A and B). This increased nucleoid clustering was absent in cardiomyocytes of BAC-TFAM mice (Fig S4A and B). Importantly, STED analysis demonstrated that the diameters were very similar to nucleoid diameters in control and BAC-TFAM mice (Fig S4C and D), consistent with our previous findings showing that a nucleoid typically contains a single copy of mtDNA (Kukat et al, 2011, 2015). The increased nucleoid clustering might thus account for less dense packing of mitochondrial cristae observed in heart tissue. In contrast to the heart, mitochondrial ultrastructure was drastically changed in skeletal muscle tissue of CAG-TFAM mice, with a strong decrease in the number of cristae and a disordered appearance (Fig 3G, right). These changes also affected the ultrastructure of the normally highly ordered and densely packed skeletal muscle fibres. Our results thus show that a balanced TFAM-to-mtDNA ratio is critical to sustain mtDNA expression and OXPHOS for normal tissue function.

\section{Tissue-specific responses to high TFAM levels}

To gain more insight into the tissue-specific responses to high TFAM levels, we proceeded to perform quantitative TMT-based proteomics of different tissues of CAG-TFAM mice. In line with our previous observations, we found a very severe depletion in the levels of OXPHOS subunits of complexes I, III, and IV and mito-ribosomal subunits in skeletal muscle, whereas the effects were milder in heart (Fig $4 \mathrm{~A}$ and B). Interestingly, whole tissue quantitative proteomics revealed very different mitochondrial responses to marked TFAM overexpression (Fig S5A). A significant fraction of all mitochondrial proteins was down-regulated in the severely affected skeletal muscle, whereas mitochondrial protein levels were maintained in the less affected heart of CAG-TFAM mice (Fig S5A). In contrast, we observed increased levels of a large fraction of all mitochondrial proteins, concomitant with stable or even mildly increased levels of OXPHOS proteins in liver of CAG-TFAM mice on a whole cell level (Figs 4A and S5A). The TFAM protein levels in liver were lower than the levels in heart or skeletal muscle (Fig 4C). Interestingly, the LONP1 protease was among the most upregulated proteins in liver of CAG-TFAM mice, but it was not increased in heart or skeletal muscle (Fig 4D). Other studies have reported that LONP1 degrades excess TFAM that is not bound to mtDNA (Matsushima et al, 2010; Lu et al, 2013). This free TFAM has been reported to be degraded by LONP1 after being marked by sitespecific phosphorylation (Lu et al, 2013). However, despite the very high TFAM levels in skeletal muscle of CAG-TFAM mice (Fig 3A and B) we did not observe increased TFAM phosphorylation by Phostag gel electrophoresis experiments (Fig S5B). A similar observation was made in liver (Fig S5C). We cannot rule out that more sensitive techniques may be required to detect post-translational modifications regulating only a subset of nucleoids.

Thus, in contrast to heart and skeletal muscle, liver appears to respond to increased TFAM levels by a compensatory response including the up-regulation of LONP1 and components of the mitochondrial transcription machinery such as the mitochondrial RNA polymerase (POLRMT) (Fig 4C and D).

Figure 3. High TFAM-to-mtDNA ratios abolish mtDNA expression in skeletal muscle.

(A) Western blot analysis of TFAM protein levels in heart and skeletal muscle whole cell lysates of CAG-TFAM (+/CAG) mice. Litter mates were used as controls (Con). Actin was used as a loading control. The asterisk indicates the lower wild-type TFAM band in control mice as opposed to the overexpression of the FLAG-tagged version of TFAM in CAG-TFAM mice. A representative image is shown ( $n=2$ independent experiments). (B) TFAM protein levels in heart and skeletal muscle whole cell lysates of CAG-TFAM animals (+/CAG) and control litter mates were quantified by densitometry and are expressed as folds of control (means \pm SEM, $\mathrm{n}=4-5$ biological replicates; $P<0.0001$ : $* * * *$, two-way ANOVA with Sidak's test for multiple comparisons). (c) Quantification of steady-state mtDNA levels in heart and skeletal muscle of CAG-TFAM (+/CAG) animals and control litter mates (Con). mtDNA levels were quantified by qPCR using specific probes against COX1 and 18S. Data are expressed as means \pm SEM ( $n=6-7$ biological replicates for heart; $n=3$ for skeletal muscle; n.S., non-significant, $P<0.0001$ : ${ }^{* * *}$, two-way ANOVA with Sidak's test for multiple comparisons). (D) Southern blot analysis of Pstl-digested mtDNA derived from heart and skeletal muscle of CAG-TFAM (+/CAG) animals and control litter mates (Con). mtDNA was quantified by radiolabeling with a specific probe against COX1, nuclear DNA was probed with 18S. A representative image is shown ( $n=3$ independent experiments). (E) Analysis of steady-state mitochondria transcript levels in heart and skeletal muscle of CAG-TFAM (+/CAG) animals and control litter mates (Con) by qRT-PCR. Mitochondrial mRNAs and tRNAs were quantified using specific mouse probes, $\beta$-2-microglobulin was used as a reference gene. ( $n=5$ biological replicates, $P<0.05$ : $* P<0.001$ : ${ }^{* * *} ; P<0.0001$ : ${ }^{* * *}$, two-way ANOVA with Sidak's test for multiple comparisons). (F) Western blot analysis of steady-state levels of respiratory chain subunits in heart and skeletal muscle mitochondrial extracts of CAG-TFAM (+/CAG) animals and control litter mates (Con). A representative image is shown ( $n=3$ independent experiments). (G) Representative images of fixed heart (left) and skeletal muscle (right) tissue from control (con) and CAG-TFAM animals at $16 \mathrm{~d}$ of age analysed by transmission electron microscopy. For each genotype, six biological replicates were analysed. Scale bars, heart: 20 (upper panels), 5 (middle), $1 \mu \mathrm{m}$ (lower panels); skeletal muscle: 10 (upper panel), 2 (middle), and $1 \mu \mathrm{m}$ (lower panel). Source data are available for this figure. 
A
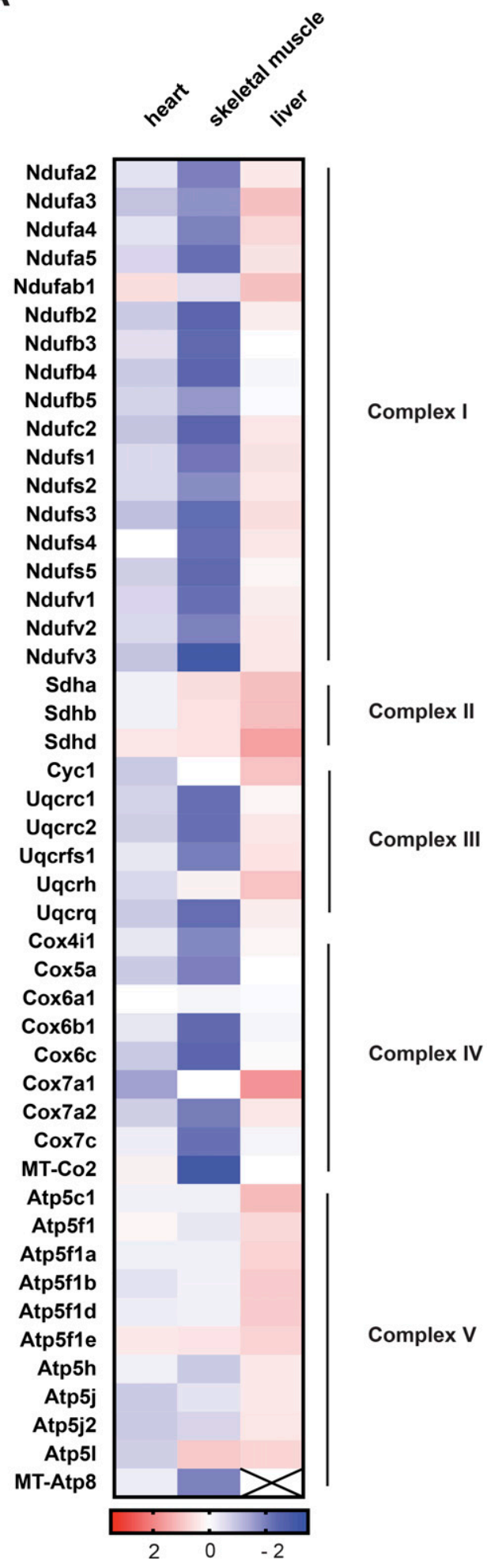

B
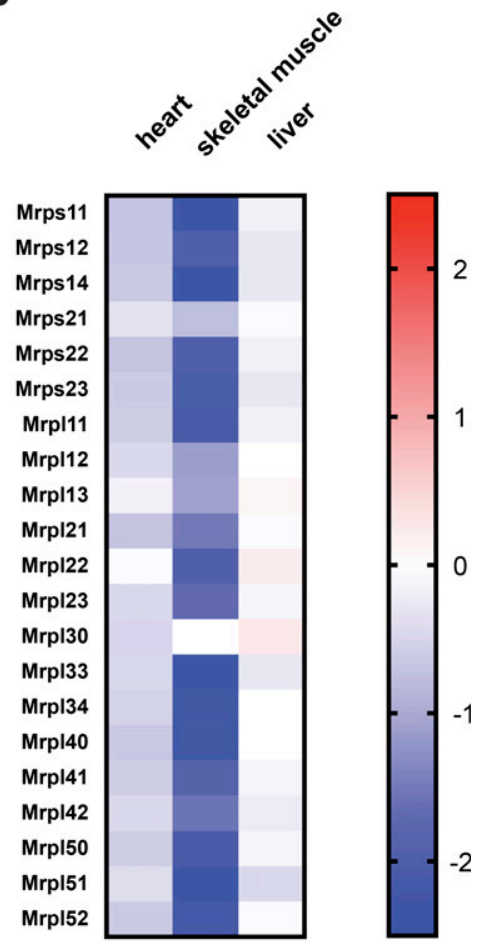

C
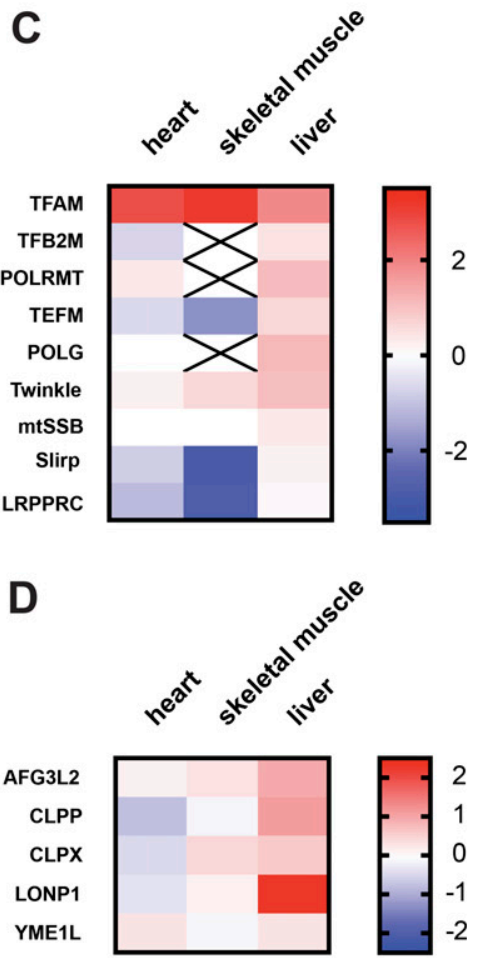

Figure 4. Tissue-specific responses to high TFAM levels.

(A, B, C, D) Heat map illustrating the $\log _{2}$ foldchange in protein levels of OXPHOS subunits (A), mitoribosomal subunits (B)

components of the mtDNA expression machinery (C), and ATP-dependent mitochondrial proteases (D) in the heart, skeletal muscle, and liver of CAG-TFAM mice compared to litter mates. Heat map: minimum $(-2)$, blue; maximum (2), red.
Mitochondrial gene expression is maintained in liver tissue

Next, we analysed the effects of the observed compensatory responses on mitochondrial gene expression in liver. Western blotting of total tissue extracts from CAG-TFAM mice confirmed the lower increase in TFAM levels in liver (2.10-fold of control, Fig $5 \mathrm{~A}$ and B). We observed no significant increase in mtDNA levels in liver (Fig $5 \mathrm{C}$ and D). The relative TFAM-to-mtDNA ratio is thus $\sim 2.41$-fold 


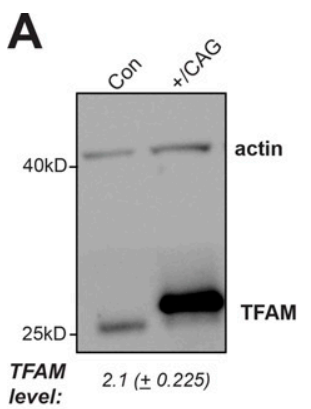

$\mathbf{F}$

sk. muscle liver

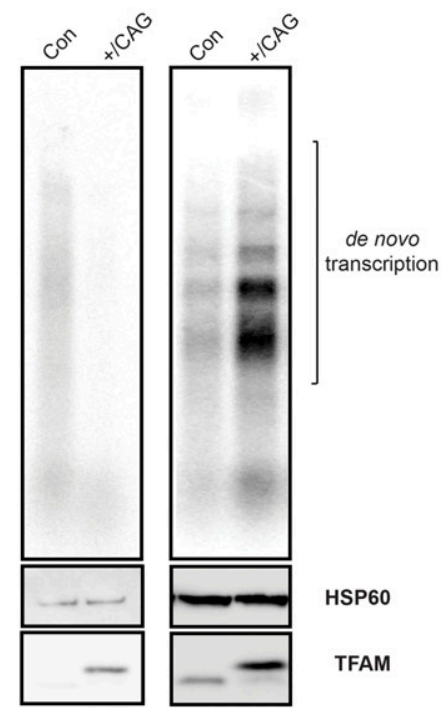

B

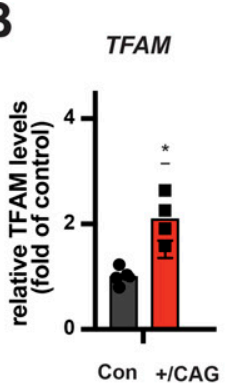

C

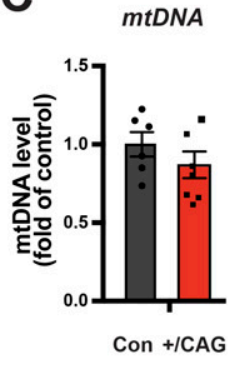

D

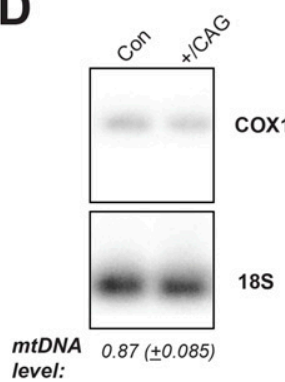

G

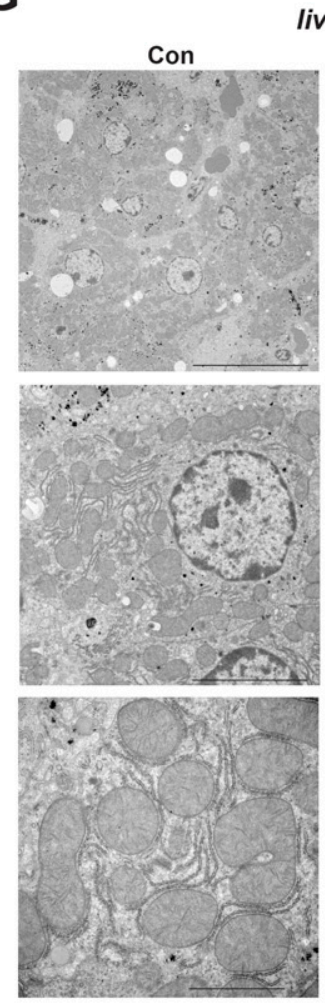

liver

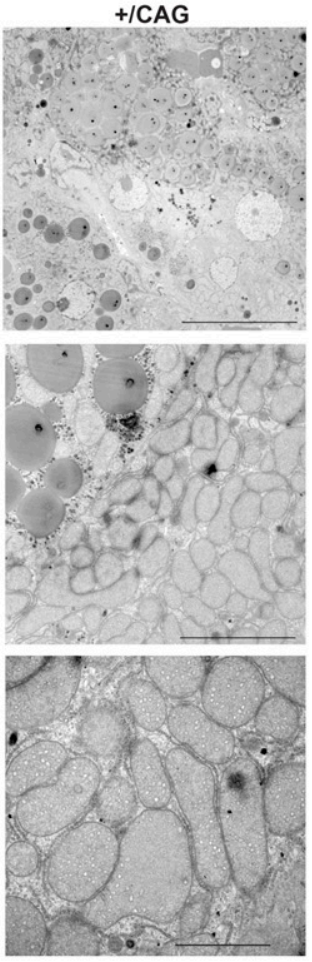

E

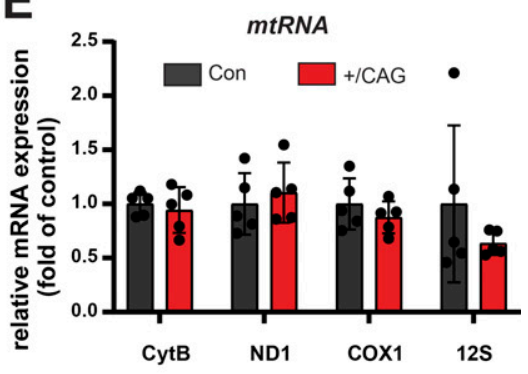

H
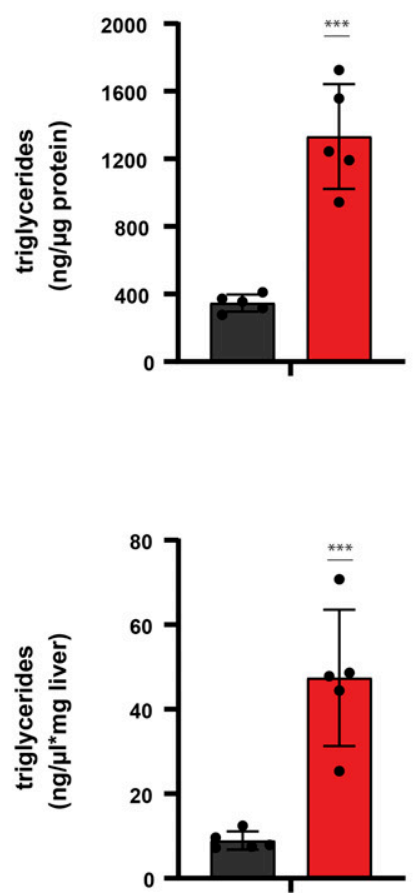

Figure 5. mtDNA expression is maintained despite high TFAM levels in liver.

(A) Western blot analysis of TFAM protein levels in liver whole cell lysates of CAG-TFAM (+/CAG) mice. Litter mates were used as controls (Con). Actin was used as a loading control. A representative image is shown ( $n=2$ independent experiments). (B) TFAM protein levels in control and CAG-TFAM animals were quantified by densitometry and are expressed as folds of control (means $\pm S E M, n=4-5$ biological replicates; $P<0.05$ : *, two-way ANOVA with Sidak's test for multiple comparisons). (C) Quantification of steady-state mtDNA levels in liver tissue of CAG-TFAM (+/CAG) animals and control litter mates (Con). mtDNA levels were quantified by qPCR using specific probes against COX1 and 18S. Data are expressed as means \pm SEM ( $n=6-7$ biological replicates). (D) Southern blot analysis of Pstl-digested mtDNA derived from heart and skeletal muscle of CAG-TFAM (+/CAG) animals and control litter mates (Con). mtDNA was quantified by radiolabeling with a specific probe against COX1, nuclear DNA was probed with 18S. A representative image is shown $(n=3$ independent experiments). (E) Analysis of steady-state mitochondrial transcript levels in liver tissue of CAG-TFAM (+/CAG) animals and control litter mates (Con) by qRT-PCR. Data are expressed as means \pm SEM ( $n=5$ biological replicates). (F) De novo RNA synthesis in skeletal muscle and liver mitochondria isolated from CAG-TFAM (+/CAG) mice and control litter mates. Mitochondria were pulse labelled for $1 \mathrm{~h}$. Mitochondrial HSP60 was used as a loading control. A representative image is shown ( $n=2-3$ independent experiments). (G) Representative images of fixed liver tissue from control (Con) and CAG-TFAM animals at $16 \mathrm{~d}$ of age analysed by transmission electron microscopy. For each genotype, six biological replicates were analysed. Scale bars, 20 (upper panels), 5 (middle), $2 \mu \mathrm{m}$ (lower panels). (H) Measurement of triglyceride content. Approximately $100 \mathrm{mg}$ of liver tissue was homogenized and triglycerides were quantified using the triglycerides quantification kit (Sigma-Aldrich). Data are expressed as means $\pm \operatorname{SEM}\left(n=5\right.$ biological replicates; $P<0.001:{ }^{* * *}$, unpaired $t$ test). Source data are available for this figure.

higher than in controls in liver of CAG-TFAM mice and this circumstance should lead to increased nucleoid compaction and reduced mtDNA expression in line with previous in vitro findings (Farge et al, 2014; Kukat et al, 2015). However, the steady-state levels of mitochondrial transcripts were normal in liver of CAG-TFAM animals as assessed by qRT-PCR (Fig 5E). Interestingly, in organello transcription assays of isolated liver mitochondria showed a much higher transcription level compared with wild-type 
mitochondria, whereas skeletal muscle mitochondria had very low de novo transcription (Fig 5F), consistent with the observed significant increase in the levels of POLRMT in liver but not in heart in our proteomic dataset (Fig 4C). It is thus apparent that an increased TFAM-to-mtDNA ratio and subsequent repression of mtDNA expression can be compensated for in some tissues, like liver. However, on an ultrastructural level we observed a marked change in mitochondrial morphology with vesicle-like cristae appearance accompanied by an increase in lipid droplets in the liver (Fig 5G). Determination of triglyceride levels relative to liver protein or tissue (Fig $5 \mathrm{H}$ ) confirmed an accumulation of lipids after strong TFAM overexpression. This points to a dysregulation of lipid metabolism, potentially as a side effect to the strong induction of mitochondrial proteases and other adaptive responses observed in liver.

To summarize, the data we present here show that a moderate increase in TFAM protein levels of 1.5-fold will not interfere with nucleoid morphology, mtDNA replication, mitochondrial gene expression and animal physiology. However, this moderate mtDNA copy number increase is sufficient to ameliorate disease phenotypes caused by heteroplasmic mtDNA mutations (Jiang et al, 2017; Filograna et al, 2019). In contrast, strong ubiquitous TFAM overexpression leads to postnatal lethality and mitochondrial dysfunction. The TFAM-to-mtDNA ratio in combination with tissue-specific regulation is critical factors to determine whether mtDNA expression will be maintained at levels sufficient to sustain OXPHOS and organ function.

\section{Discussion}

TFAM is the major structural protein of the mammalian nucleoid, covering mtDNA with a ratio of 1 TFAM molecule per 16-17 bp of mtDNA (Alam et al, 2003; Kukat et al, 2011). Importantly, TFAM is sufficient to fully compact mtDNA into nucleoids by a series of events starting with single TFAM molecules cooperatively binding to mtDNA in patches (Kaufman et al, 2007; Farge et al, 2014; Kukat et al, 2015). TFAM compacts mtDNA by cross-strand binding and loop formation, thus condensing the mammalian mtDNA with a contour length of $\sim 5 \mu \mathrm{m}$ into a nucleoid structure with a diameter of $\sim 100 \mathrm{~nm}$ (Brown et al, 2011; Kukat et al, 2011). For comparison, an even higher degree of DNA compaction is observed in the nucleus, especially in the condensed states of mammalian chromosomes. Wrapping of DNA around the histone octamer globally conceals nuclear promoters and makes them inaccessible to the preinitiation complex, rendering the core nucleosome a general nuclear gene repressor (Lorch et al, 1987; Han \& Grunstein, 1988). Nuclear transcription is thus shut off by default and a complex machinery acts to specifically activate gene expression, for example, by recruitment of the transcription initiation machinery, binding of transcriptional activators, histone remodelling, and addition of posttranslational histone modifications (Kornberg \& Lorch, 2020).

In contrast to nuclear transcription, mtDNA transcription may be constitutively active. Quantitative assessment of mtDNA transcription in wild-type mouse embryonic fibroblasts has shown that the vast majority of nucleoids are transcribed (Ramos et al, 2019). Transcripts from mtDNA form mitochondrial RNA granules next to almost all nucleoids as determined by FISH analyses of individual transcripts or BrU labelling of nascent transcripts (Ramos et al, 2019). It is thus important to recognize that regulation of mitochondrial gene expression needs to ensure promoter-specific initiation of mtDNA transcription. Besides its role in mtDNA compaction (Kaufman et al, 2007; Kukat et al, 2011, 2015), TFAM also binds specifically to the mtDNA promoters and is critical for recruiting POLRMT and TFB2M for initiation of transcription (Hillen et al, 2017) (Fig 6). Although POLRMT on its own is poor at melting doublestranded DNA (dsDNA), it can initiate transcription when binding to single-stranded (sSDNA) in vitro (Wanrooij et al, 2008). Transient strand-separation of mtDNA may result from mtDNA replication or supercoiling tension, but typically, sSDNA is covered by the mitochondrial single-stranded binding protein (mtSSB) and therefore not accessible to binding by POLRMT and initiation of unspecific transcription (Fusté et al, 2010) (Fig 6). In line with this, there is a drastic increase in unspecific transcription initiation of mtDNA in vivo in a conditional mouse knockout model lacking mtSSB in heart (Jiang et al, 2021). The promoter-specific initiation of mtDNA transcription is thus dependent on both TFAM and mtSSB.

Experiments with in vitro reconstituted mitochondrial nucleoids have shown that the TFAM-to-mtDNA ratio determines whether the nucleoid is in an open (active) or a compacted (inactive) state (Kaufman et al, 2007; Farge et al, 2014; Kukat et al, 2015). Variations in local TFAM protein levels may therefore shift the equilibrium between open and inactive nucleoids and thereby control mtDNA gene expression at the level of compaction. We show here that TFAM can also serve as a general repressor of mtDNA gene expression in vivo. We observed a substantial decrease in mitochondrial gene expression in the skeletal muscle of CAG-TFAM mice where the TFAM-to-mtDNA ratio vastly exceeds the ratio found in normal skeletal muscle. Because of the increased TFAM protein levels, the equilibrium likely shifts and may force the nucleoids to adapt a hyper-packaged state which abolishes mitochondrial gene expression (Fig 6). In heart sections of CAG-TFAM mice, we observed an increase in the number of nucleoids consistent with the increased mtDNA copy number. The nucleoids of CAG-TFAM mice were of similar size as nucleoids of control or BAC-TFAM mice, although they formed extensive clusters. Because of technical challenges, we could not directly address nucleoid size by STED microscopy of tissue sections from skeletal muscle and liver. Still, oversaturation of the system with TFAM is likely the basis for the observed skeletal muscle phenotype in CAG-TFAM mice. In agreement with this model, forced expression of Abf2p, the TFAM homologue in budding yeast, leads to eventual loss of mtDNA due to exclusion of replication factors by excessive nucleoid compaction (Zelenaya-Troitskaya et al, 1998). However, increased mtDNA copy number under respiring growth conditions resulted in a more balanced Abf2p-to-mtDNA ratio and more open, transcriptionally active nucleoids in the budding yeast (Kucej et al, 2008). Here, we observe markedly increased mtDNA levels in heart tissue of CAGTFAM mice after high levels of TFAM expression. This leads to a far more balanced TFAM-to-mtDNA ratio in heart, which allows for continued mtDNA expression. The postnatal development of cardiomyocytes involves substantial mtDNA replication during the first 4 wk of postnatal life (Ramos et al, 2019) which may explain the observed increase in mtDNA levels in heart. However, we observe 


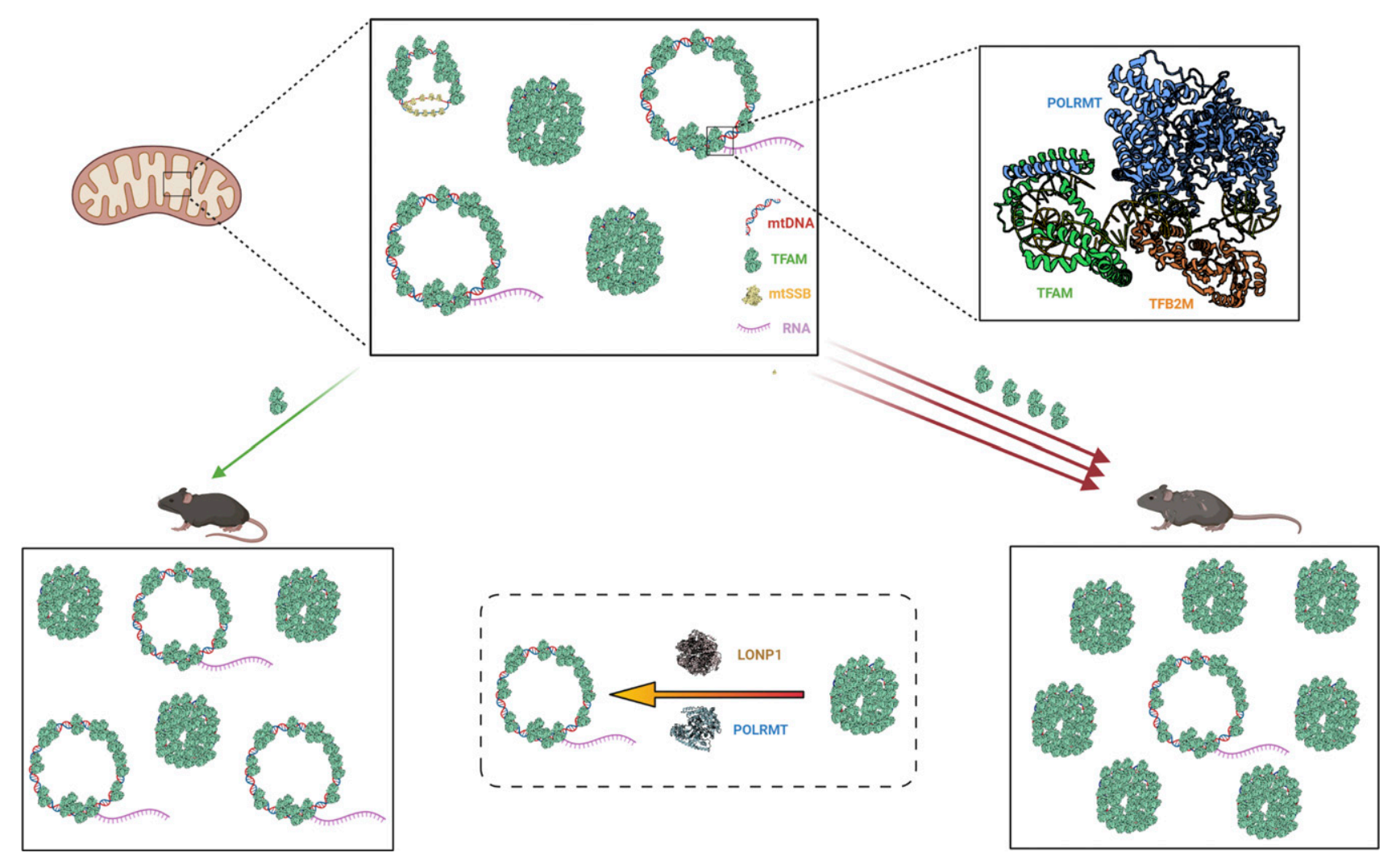

Figure 6. Regulation of mtDNA expression by TFAM levels in vivo.

An overview of the proposed regulation of mtDNA expression by TFAM-induced compaction of mitochondrial nucleoids. Open and compacted mitochondrial nucleoids are schematically depicted as circles. The other icons are explained in the figure. This figure was generated using Biorender.com (PDB entries: 3SPA, 7KSM, 2DUD, 6ERP, 3TMM) (Ngo et al, 2011; Ringel et al, 2011; Hillen et al, 2017).

milder adverse effects of TFAM overexpression in the heart and we cannot rule out that more detrimental effects in heart may occur with increasing age despite initial compensation mechanisms (Ghazal et al, 2021). TFAM-to-mtDNA ratios also vary between the different stages of Xenopus oocyte development (Shen \& Bogenhagen, 2001), thus TFAM-mediated repression may represent an important, conserved mechanism that controls mtDNA expression in response to the metabolic needs during development and for appropriate function of differentiated tissues.

Our data indicate that regulatory mechanisms may counteract TFAM-induced mitochondrial gene repression in certain tissues. We observed a marked increase in LONP1 levels in the liver of CAGTFAM mice. In contrast to skeletal muscle, TFAM protein levels were lower and the TFAM-to-mtDNA ratio was more balanced in liver, which may explain the near-normal mitochondrial transcript levels. The TFAM-to-mtDNA ratio observed in liver should be sufficient to repress mtDNA transcription according to in vitro findings (Farge et al, 2014), but the action of LONP1 in vivo may lead to local changes in TFAM levels that may result in decreased compaction of the nucleoid at the promoter regions, which may allow recruitment of POLRMT and TFB2M for initiation of transcription. We observed an induction of both LONP1 and POLRMT expression in liver, which may keep a larger fraction of nucleoids in an open, active state to allow promoter-specific transcription initiation. The tissue-specific induction of the LONP1 protease in liver, but not in skeletal muscle or heart, points to the existence of cell-type-specific responses regulating TFAM levels. However, this adaptation may come with a cost as we observed accumulation of triglycerides in the liver, which points to dysregulation of lipid metabolism, possibly as a consequence of the up-regulation of mitochondrial proteases interfering with metabolic pathways.

Our data highlight the importance of maintaining TFAM-tomtDNA ratios within a certain physiological interval to allow proper regulation of mtDNA transcription. Local changes in TFAM levels may provide an important mechanism that controls the switch between repression and activation of mtDNA gene expression by regulation of promoter-specific transcription initiation. Possible treatment strategies to counteract mitochondrial dysfunction by induction mitochondrial biogenesis, for example, by activation of PGC1 $\alpha$, or selective TFAM overexpression must take into account the resulting TFAM-to-mtDNA ratios. Based on the findings presented here, we conclude that modulation of TFAM levels to increase mtDNA copy number by 1.5 -fold provides a safe intervention, whereas strong overexpression of TFAM may result in excessive repression of mitochondrial gene expression, nucleoid clustering and ultrastructural changes of mitochondria in different tissues. We thus conclude that the TFAM-to-mtDNA ratio provides an essential mechanism that control mtDNA gene expression in vivo and that this ratio must be maintained or proper function oxidative phosphorylation. 


\section{Materials and Methods}

\section{Animal models and housing}

We generated BAC-TFAM mice as previously described (Jiang et al, 2017). Founder mice carrying Tfam BAC DNA were identified by Pvul restriction analysis of mouse genomic DNA. Germ line transmission was established from different founder mice and the offspring was kept as heterozygous stocks by breeding to C57Bl/6N mice. Three different mouse lines (BAC TG 188, TG 137 and TG 91) corresponding to three different founders were kept for molecular analysis.

The plasmid construction was performed as previous described (Sterky et al, 2011). In brief, a mouse Tfam CDS sequence without a stop codon was cloned and a flag sequence was added at its $C$ terminus. This sequence was cloned into the Serca plasmid backbone containing CAG promoter-loxP-Neo-Westfal Stop cassette-loxP sites (kind gift from $T$ Wunderlich). The targeting vector was linearized and electroporated into mouse embryonic stem (ES) cells. Southern blotting was used to identify the positive colonies from transformed ES cell lines, which were used for blastocyst injection, and germ line transmission was obtained by mating chimeric mice to $\mathrm{C} 57 \mathrm{BL} / 6 \mathrm{~N}$ mice.

All mice used in this study had an inbred C57Bl/6N background and were housed in standard individually ventilated cages in a $12 \mathrm{~h}$ light/dark cycle in controlled environmental conditions $\left(21^{\circ} \mathrm{C} \pm 2^{\circ} \mathrm{C}\right.$, $50 \%+10 \%$ relative humidity). Mice were fed a normal chow diet (ssniff) and water ad libitum. The study was approved by the Landesamt für Natur, Umwelt und Verbraucherschutz, NordrheinWestfalen, Germany (reference numbers 84-02.04.2015.A103, 8402.50.15.004 and 84-02.04.2016.A420) and performed in accordance with the recommendations and guidelines of the Federation of European Laboratory Animal Science Associations.

\section{Analysis of energy homeostasis}

Energy homeostasis and activity of BAC-TFAM animals and agematched wild-type mice was measured at the age of 10 and 52 wk using metabolic cages (Phenomaster; TSE systems). Before the actual experiments, animals were acclimatized to the different housing conditions in training cages for 3-4 $\mathrm{d}$. Mice were housed in metabolic cages for 3-4 $d$ with data being collected for a time span of $48 \mathrm{~h}$. Differences in $\mathrm{O}_{2}$ and $\mathrm{CO}_{2}$ levels were measured and were used to calculate $\mathrm{O}_{2}$ consumption, $\mathrm{CO}_{2}$ production, respiratory exchange rate, and energy expenditure (heat). Disruption of light beams simultaneously documented animal activity.

\section{Isolation of total protein and mitochondria from tissues}

Animals were euthanized by cervical dislocation, and isolated tissues were cleaned and directly snap-frozen in liquid nitrogen. Total proteins were extracted from ground tissue powder using $2 \times$ SDS-sample buffer (100 mM Tris, pH 6.8, 4\% SDS, 20\% glycerol, and 200 mM DTT) supplemented with complete protease and phosphatase inhibitor cocktail (Roche). The protein concentration was determined using the RCDC assay (Bio-Rad) and BSA as a standard.
Mitochondria were isolated from mouse tissues using differential centrifugation. Briefly, fresh tissues were cut, washed with ice-cold PBS and homogenized in standard mitochondrial isolation buffer containing $320 \mathrm{mM}$ sucrose, $10 \mathrm{mM}$ Tris- $\mathrm{HCl}$, and $1 \mathrm{mM}$ EDTA by using a Potter $\mathrm{S}$ pestle (Sartorius). The homogenate was centrifuged at $1,000 \mathrm{~g}$ for $10 \mathrm{~min}$ at $4^{\circ} \mathrm{C}$. The supernatant was collected and centrifuged at $10,000 \mathrm{~g}$ for $10 \mathrm{~min}$ at $4^{\circ} \mathrm{C}$. Resulting crude mitochondrial pellets were resuspended in mitochondrial isolation buffer supplemented with complete protease inhibitor cocktail (Roche). Protein concentration was determined using the Bradford method (Sigma-Aldrich) and BSA as a standard.

\section{Gel electrophoresis and Western blotting}

For standard gel electrophoresis, protein samples (30 $\mu \mathrm{g} /$ lane) were mixed with $2 \times$ NuPAGE LDS sample buffer supplemented with $200 \mathrm{mM}$ DTT and resolved using commercially available $10 \%$ or 4-12\% NuPAGE Bis-Tris gels and MOPS or MES buffer (Invitrogen) including protein standards (Spectra Multicolor Broad Range; Thermo Fisher Scientific). Proteins were transferred on nitrocellulose membranes using wet tank blotting $(25 \mathrm{mM}$ Tris, $192 \mathrm{mM}$ glycine, and $20 \%$ methanol) at $4^{\circ} \mathrm{C}$ for $2 \mathrm{~h}$ at $400 \mathrm{~mA}$ or overnight at $80 \mathrm{~mA}$.

For detection of TFAM phosphorylation, the tissues were lysed in $20 \mathrm{mM}$ Tris- $\mathrm{HCl}, 150 \mathrm{mM} \mathrm{NaCl}, 1 \mathrm{mM}$ EDTA, 1 mM EGTA, 1\% Triton $\mathrm{X}-100$, and protease inhibitors. $100 \mu \mathrm{g}$ of whole tissue lysate were treated with or without $\lambda$ phosphatase (NEB) according to the manufacturer's instructions. Samples were precipitated using TCA and protein pellets were resuspended in $2 \times$ SDS sample buffer supplemented with $\beta$-mercaptoethanol. Samples were boiled for 5 min before loading on $\mathrm{Zn}^{2+}$ Phostag gels using a neutral-pH Bis-Tris buffering system (15 $\mu \mathrm{g} /$ lane) (Kinoshita \& Kinoshita-Kikuta, 2011). Briefly, 10\% polyacrylamide Bis-Tris gels were prepared with the addition of both Phostag acrylamide (50 $\mu \mathrm{M}$ final) and $\mathrm{ZnCl}_{2}(100 \mu \mathrm{M}$ final) and run in buffer containing $100 \mathrm{mM} \mathrm{MOPS}, 100 \mathrm{mM}$ Tris, $5 \mathrm{mM}$ sodium bisulfite, and $0.10 \%$ SDS. Phostag gels were soaked three times in transfer buffer containing 5 mM EDTA for 10 min to remove $\mathrm{Zn}^{2+}$ before wet transfer overnight.

For standard and Phostag PAGE, membranes were blocked in $5 \%$ milk-1× Tris-buffered saline-0.1\% Tween 20 (TBST) for at least $1 \mathrm{~h}$ at room temperature. Membranes were subsequently incubated with primary antibodies diluted in $5 \%$ milk-TBST overnight at $4^{\circ} \mathrm{C}$, washed in TBST and incubated with HRP-conjugated secondary antibodies for $2 \mathrm{~h}$ at room temperature. After washing with TBST, immunodetection was performed by enhanced chemiluminescence (GE Healthcare) using either photo film or the Fujifilm LAS $400 \mathrm{im}$ aging system (Fujifilm). The following antibodies were used: rabbit polyclonal anti-TFAM (Abcam), mouse monoclonal anti-actin (Abcam), Total OXPHOS Rodent Western Blotting antibody cocktail (Abcam), mouse monoclonal anti-tubulin (Sigma-Aldrich), rabbit anti-Tubulin (Cell Signaling), rabbit anti-vinculin (Abcam), mouse monoclonal anti-UQCRFS1/RISP (Abcam), mouse monoclonal anti-COX5a (Invitrogen/ThermoFisher Scientific), mouse monoclonal anti-VDAC (Millipore), rabbit polyclonal anti-ACOT2 (Proteintech), mouse monoclonal anti-cytochrome C (Abcam), rabbit anti-HSP60 (Cell Signaling), sheep anti-mouse IgG (GE Healthcare), and donkey anti-rabbit (GE Healthcare). For densitometry of 
protein levels, intensity of protein bands was analysed using either the MultiGauge Software (Fuji) on LAS imaging files or ImageJ on scanned photo film.

\section{DNA-isolation and determination of mtDNA levels by qPCR}

Total DNA was extracted and purified using the Puregene Core A Kit (QIAGEN) following the manufacturer's instructions including RNAse treatment. The purity and quantity of DNA were evaluated with the NanoDrop 2000 (Thermo Fisher Scientific) and $5 \mathrm{ng} / \mu \mathrm{l}$ DNA were analysed by qPCR. qPCR was carried out using the Taqman $2 \times$ Universal PCR mastermix, No Amperase UNG (Applied Biosystems), and commercially available Taqman assay probes for mouse mitochondrial (COX1, Mm04225243_g1) and nuclear DNA (18S, Hs99999901_s1).

\section{Southern blotting}

$2 \mu \mathrm{g}$ of total DNA were digested with Pstl restriction enzyme overnight at $37^{\circ} \mathrm{C}$. Digested DNA was precipitated with ethanol, resuspended in water and DNA fragments were separated overnight by $0.6-0.8 \%$ agarose gel electrophoresis. Southern blotting to nitrocellulose membranes (Hybond-N+; GE healthcare) was carried out as described before (Kauppila et al, 2018). Hybridisation was carried out using radiolabelled probes against mouse COX1 and $18 \mathrm{~S}$ to detect mitochondrial and nuclear DNA, respectively.

\section{RNA-isolation, Northern blotting, cDNA synthesis, and qRT-PCR}

Total RNA was isolated from snap-frozen tissue samples using TRIzol Reagent (Thermo Fisher Scientific) following the manufacturer's instructions and subsequently DNAse-treated (TURBO DNAfree kit; Thermo Fisher Scientific). If only intended for Northern blotting, the RNA pellet was directly resuspended in deionized formamide. Alternatively, total RNA was isolated using the Directzol RNA Miniprep Kit (ZymoResearch) including the DNAse digestion step. Purity and quantity of RNA were evaluated with the NanoDrop 2000 (Thermo Fisher Scientific). Northern blot analysis was carried out as previously described (Kauppila et al, 2018). Briefly, $2 \mu \mathrm{g}$ of RNA were separated on $1.2 \%$ paraformaldehyde-agarose gel in MOPS running buffer and transferred to nitrocellulose membranes (Hybond- $\mathrm{N}+$ ) by capillary transfer. Detection of mitochondrial mRNAs and rRNAs was carried out using ${ }^{32} \mathrm{P}$-dCTP-labeled probes, mitochondrial tRNAs were detected using specific oligonucleotides labelled with $y^{-32}$ P-ATP. 18S was hybridised as a loading control. cDNA was synthesized using the High-Capacity RNA-to-cDNA Kit (Applied Biosystems). qRT-PCR was carried out using the Taqman $2 \times$ Universal PCR mastermix, No Amperase UNG (Applied Biosystems) and commercially available Taqman Assay probes for mouse mitochondrial transcripts (CytB, Mm04225271_g1; ND1, Mm04225274_s1; COX1, Mm04225243_g1; 12S, Mm04260177_s1; Life Technologies). Transcript quantities were normalized to $\beta$-2-microglobulin used as a reference gene transcript (Mm00437762_m1; Life Technologies).

\section{In organello transcription}

Mitochondria were isolated from fresh heart and liver tissue by differential centrifugation $(1 \times 1,000 \mathrm{~g}, 1 \times 10,000 \mathrm{~g})$. For in organello transcription experiments, freshly purified mitochondria (500 $\mu \mathrm{g}$ ) were washed three times in incubation buffer $(25 \mathrm{mM}$ sucrose, 75 mM sorbitol, $10 \mathrm{mM}$ Tris- $\mathrm{HCl}, 10 \mathrm{mM} \mathrm{K}{ }_{2} \mathrm{HPO}_{4}, 100 \mathrm{mM} \mathrm{KCl}, 0.05 \mathrm{mM}$ EDTA, $1 \mathrm{mM}$ ADP , $5 \mathrm{mM} \mathrm{MgCl}_{2}, 10 \mathrm{mM}$ glutamate, $2.5 \mathrm{mM}$ malate, and 1 $\mathrm{mg} / \mathrm{ml} \mathrm{BSA}, \mathrm{pH}$ 7.4). Washed mitochondria were resuspended in 500 $\mu \mathrm{l}$ of incubation buffer and supplemented with $50 \mu \mathrm{Ci}$ of $\alpha^{-32} \mathrm{P}$-UTP (Hartmann Analytic). Samples were incubated at $37^{\circ} \mathrm{C}$ for $1 \mathrm{~h}$. Afterwards, mitochondria were pelleted, resuspended in incubation buffer containing $0.2 \mathrm{mM}$ UTP and incubated for $10 \mathrm{~min}$ at $37^{\circ} \mathrm{C}$. Mitochondria were subsequently washed twice in cold wash buffer (10\% glycerol, $0.15 \mathrm{mM} \mathrm{MgCl}_{2}$, and $10 \mathrm{mM}$ Tris- $\mathrm{HCl}, \mathrm{pH}$ 6.8). An aliquot of the mitochondria $(10 \mu \mathrm{l})$ was taken as a loading control. Then, RNA was extracted using TRIzol (Ambion) following the manufacturer's recommendations and precipitated overnight at $-20^{\circ} \mathrm{C}$. The purified RNA was loaded onto a formaldehyde-agarose gel and blotted as a northern blot. The membrane (Hybond- $\mathrm{N}+$; GE Healthcare) was exposed to a phosphorimager screen. Loading controls were run on $10 \%$ NuPage gels using MOPS buffer and equal loading was assessed by immunoblotting against HSP60.

\section{Quantitative proteomics}

Sample preparation from $10 \mathrm{mg}$ of grinded tissue powder was performed as described (Busch et al, 2019) with slight modifications: tryptic peptides were eluted from STAGE tips with $40 \%$ acetonitrile (ACN) $0.1 \%$ formic acid (FA). $4 \mu \mathrm{g}$ of desalted peptides were labelled with TMTs (TMT10plex, Cat. no 90110; Thermo Fisher Scientific) using a 1:20 ratio of peptides to TMT reagent. All 10 samples per time point were labelled in one TMT batch. TMT labelling was carried out according to manufacturer's instruction with the following changes: $0.8 \mathrm{mg}$ of TMT reagent was re-suspended with $70 \mu \mathrm{l}$ of anhydrous ACN, dried peptides were reconstituted in $9 \mu \mathrm{l} 0.1 \mathrm{M}$ Tetraethylammoniumbromid (TEAB) to which $7 \mu \mathrm{l}$ TMT reagent in ACN was added to a final ACN concentration of $43.75 \%$, after 60 min of incubation the reaction was quenched with $2 \mu \mathrm{l} 5 \%$ hydroxylamine. Labelled peptides were pooled, dried, resuspended in $200 \mu \mathrm{l} 0.1 \%$ FA, split into two samples, and desalted using home-made C18 STAGE tips (Rappsilber et al, 2003). One of the two halves was fractionated on a $1 \times 150$ mm ACQUITY column, packed with $130 \AA$, 1.7 $\mu \mathrm{m}$ C18 particles (Cat. no. SKU: 186006935; Waters), using an Ultimate 3000 UHPLC (Thermo Fisher Scientific). Peptides were separated at a flow of $30 \mu \mathrm{l} / \mathrm{min}$ with a 96 min segmented gradient from $1 \%$ to $50 \%$ buffer B for 85 min and from $50 \%$ to $95 \%$ buffer B for 3 min, followed by 8 min of $95 \%$ buffer B; buffer A was $5 \%$ ACN, $10 \mathrm{mM}$ ammonium bicarbonate (ABC), buffer B was $80 \%$ ACN, $10 \mathrm{mM} \mathrm{ABC}$. Fractions were collected every $3 \mathrm{~min}$, and fractions were pooled in two passed $(1+17,2+18 \ldots$ etc. $)$ and dried in a vacuum centrifuge (Eppendorf). Dried fractions were resuspended in $0.1 \% \mathrm{FA}$ separated on a $50 \mathrm{~cm}, 75 \mu \mathrm{m}$ Acclaim PepMap column (Cat. no 164942; Thermo Fisher Scientific) and analysed on a Orbitrap Lumos Tribrid mass spectrometer (Thermo Fisher Scientific) equipped with a field asymmetric ion mobility spectrometry (FAIMS) device (Thermo Fisher Scientific) that was operated in two compensation voltages, -50 and $-70 \mathrm{~V}$. Alternatively, peptides were separated on a $25 \mathrm{~cm}$, $75 \mu \mathrm{m}$ PicoFrit column (New Objective) packed with $1.9 \mu \mathrm{m}$ ReproSil-Pur media (Dr. Maisch) and analysed on an Orbitrap Fusion Tribrid mass spectrometer (Thermo Fisher Scientific). Synchronous 
precursor selection based MS3 was used for TMT reporter ion signal measurements. Peptide separations were performed on an EASYnLC1200 using a 90 min linear gradient from 6 to 31\% buffer; buffer A was $0.1 \%$ FA, buffer B was $0.1 \%$ FA, $80 \%$ ACN. The analytical column was operated at $50^{\circ} \mathrm{C}$. Raw files were split based on the FAIMS compensation voltage using FreeStyle (v. 1.6; Thermo Fisher Scientific).

Proteomics data were analysed using MaxQuant (Cox \& Mann, 2008) (version 1.6.10.43). Raw proteomics data was searched against the mouse proteome database from Uniprot, downloaded in September 2018. The isotope purity correction factors, provided by the manufacturer, were included in the analysis. Differential expression analysis was performed using limma (Ritchie et al, 2015) in $R$ ( $R$ Core Team, 2018). The raw data, database search results, and the data analysis workflow and results were deposited to the ProteomeXchange Consortium via the PRIDE partner repository (Perez-Riverol et al, 2019) with the dataset identifier PXD023050.

\section{Quantification of triglycerides}

Approximately $100 \mathrm{mg}$ of liver tissue was homogenized in a $1 \mathrm{ml}$ solution of $5 \%$ IGEPAL CA-630 (Sigma-Aldrich). The homogenate was incubated at $90^{\circ} \mathrm{C}$ for $5 \mathrm{~min}$, then cooled to room temperature. The heating-cooling cycle was repeated to solubilize all triglycerides. Samples were centrifuged at top speed for $2 \mathrm{~min}$, and the supernatant was collected. The samples were diluted 10 -fold with water before the quantification, then 5-30 $\mu \mathrm{l}$ of the samples were used with the triglycerides quantification kit (Sigma-Aldrich) following the manufacturer's instructions.

\section{COX/SDH staining}

COX/SDH double staining was performed as previously described (Matic et al, 2018). Briefly, fresh heart and skeletal muscle tissues were dissected and immediately frozen in isopentane chilled with liquid nitrogen. Tissues were further cryo-sectioned into sections (10 $\mu \mathrm{m}$ for skeletal muscle and $7 \mu \mathrm{m}$ for heart), mounted on slides and left to air dry briefly. Freshly prepared buffer A $(0.8 \mathrm{ml}$ of $5 \mathrm{mM}$ 3,3'-diaminobenzidine tetrahydrochloride, $0.2 \mathrm{ml}$ of $500 \mu \mathrm{M}$ cytochrome $\mathrm{c}$, and $10 \mu \mathrm{l}$ of catalase) was added to the slides. After incubation for $60 \mathrm{~min}$ at $37^{\circ} \mathrm{C}$, slides were washed three times by 0.1 $\mathrm{M}$ phosphate buffered saline, $\mathrm{pH}$ 7.0. Then freshly prepared buffer $\mathrm{B}$ $(0.8 \mathrm{ml} 1.875 \mathrm{mM}$ of nitroblue tetrazolium, $0.1 \mathrm{ml} 1.3 \mathrm{M}$ of sodium succinate, $0.1 \mathrm{ml} 2.0 \mathrm{mM}$ phenazine methosulphate, and $10 \mu \mathrm{l}$ of 100 $\mathrm{mM}$ sodium azide) was applied and incubated for $30 \mathrm{~min}$ at $37^{\circ} \mathrm{C}$. Slides were washed three times with $0.1 \mathrm{M}$ phosphate buffered saline, pH 7.0, dehydrated and mounted for bright-field microscopy.

\section{Transmission electron microscopy}

Liver, skeletal muscle and heart tissue were cut into small pieces and fixed in $2.5 \%$ glutaraldehyde, $1 \%$ paraformaldehyde, and $0.1 \mathrm{M}$ phosphate buffer, $\mathrm{pH} 7.4$ at room temperature for $1 \mathrm{~h}$, followed by 24 $\mathrm{h}$ at $4^{\circ} \mathrm{C}$. After the fixation, the specimens were rinsed in a buffer containing $0.1 \mathrm{M}$ sodium phosphate and subsequently post-fixed in $2 \%$ osmium tetroxide at $4^{\circ} \mathrm{C}$ for $2 \mathrm{~h}$. The specimens were then stepwise dehydrated in ethanol followed by acetone and finally embedded in LX-112 (Ladd Research Industries). Ultra-thin sections
(60-80 nm) from longitudinal parts were cut and examined in a Hitachi HT7700 (Hitachi High-Tech) at 80 kV. Equipped with a 2kx2k Veleta CCD camera (OSIS). Digital images at a final magnification of $2,500 \times, 5,000 \times, 10,000 \times, 20,000 \times$, and 40,000× were randomly acquired from sections of the tissues.

\section{gSTED analysis of nucleoids in heart tissue}

\section{Immunohistochemistry}

Freshly isolated hearts were fixed in 4\% PFA, cryopreserved in 30\% sucrose and frozen in optimal cutting temperature compound (OCT). 10- $\mu$ m-thick cryosections were cut and air-dried before proceeding with fluorescent immunohistochemistry. Sections were permeabilized in $0.5 \%$ Triton X-100/PBS and unspecific binding of antibodies was prevented by incubation in blocking buffer (3\% BSA) PBS). The following primary antibodies in blocking buffer were then applied overnight at $+4^{\circ} \mathrm{C}$ : mouse IgM anti-DNA (Progen), rabbit anti-TOM20 (Santa Cruz). After washing, the following secondary antibodies were then applied: goat anti-mouse IgM Alexa 594, and donkey anti-rabbit Alexa 488. Nuclei were counterstained with DAPI.

\section{Imaging}

Imaging was performed with a Leica TCS SP8 gated STED (gSTED) microscope, with a white light laser and a 93× objective lens (HC PL APO CS2 93× GLYC, NA 1.30). For confocal images of mitochondria (TOM20) and DNA, Z-stacks were taken by exciting the fluorophores at 488 and $594 \mathrm{~nm}$, respectively, and Hybrid detectors collected fluorescent signals. STED images of DNA channel were obtained with a 775-nm depletion laser. 2D confocal and gSTED images were acquired sequentially with the optical zoom set to obtain a voxel size of $17 \times 17 \mathrm{~nm}$. Excitation was provided at $594 \mathrm{~nm}$ and Hybrid detectors collected signal. Gating between 0.3 and 6 ns was applied. Performance of the microscope and optimal depletion laser power were tested as previously described (Nicholls et al, 2018).

\section{Image processing}

Raw images were first deconvolved with the Huygens software. Image panels were created with Photoshop (Adobe); no digital manipulation was applied, except for adjustment of brightness and contrast.

\section{Data Availability}

All relevant data generated and analysed are included in the article. For the proteomic data, the raw data, database search results, and the data analysis workflow and results were deposited to the ProteomeXchange Consortium via the PRIDE partner repository with the dataset identifier PXD023050. Source data are provided with this article. Further requests should be directed to the corresponding authors.

\section{Supplementary Information}

Supplementary Information is available at https://doi.org/10.26508/lsa. 202101034. 


\section{Acknowledgements}

Work described here was supported by the Max Planck Society, the Swedish Research Council (2015-00418 to N-G Larsson), the Swedish Cancer Foundation (to N-G Larsson), the Knut and Alice Wallenberg foundation (to $\mathrm{N}-\mathrm{G}$ Larsson), the Deutsche Forschungsgemeinschaft (SFB1218/A06 to N-G Larsson), European Research Council (Advanced grant 2016-741366 to N-G Larsson), and the ALF agreement (agreement between the Swedish state and some county councils on cooperation on basic education of doctors, medical research, and the development of health care) to N-G Larsson (SLL2018.0471). M Jiang was supported by the Westlake Education Foundation. E Motori was supported by the SFB1218 Advanced PostDoc Grant. We thank Nadine Hochhard for excellent technical assistance. We thank Avan Taha and Xinping Li from the Proteomic Core Facility and Martin Purrio of the Phenotyping Core Facility for support. We thank the Karolinska Center for Transgene Technologies for technical support in generating CAG-TFAM and the TFAM BAC mouse lines. The serca backbone plasmid was a kind gift from T Wunderlich. We thank the FACS \& Imaging Core Facility at the Max Planck Institute for Biology of Ageing and the CECAD Imaging Facility. We thank Lars Haag and the Department of Laboratory Medicine at Karolinska for assistance in transmission electron microscopy. Fig 6 was created using Biorender.com using the protein data bank entries 3SPA, 7KSM, 6DUD, 6ERP, and 2TMM.

\section{Author Contributions}

NA Bonekamp: conceptualization, data curation, formal analysis, supervision, validation, investigation, visualization, methodology, project administration, and writing-original draft, review, and editing. M Jiang: data curation, investigation, and methodology. E Motori: data curation, formal analysis, investigation, and methodology. R Garcia Villegas: data curation, formal analysis, and investigation. C Koolmeister: methodology.

I Atanassov: data curation, formal analysis, and investigation.

A Mesaros: formal analysis and investigation.

CB Park: methodology.

N-G Larsson: conceptualization, resources, supervision, funding acquisition, project administration, and writing-original draft, review, and editing.

\section{Conflict of Interest Statement}

$\mathrm{N}-\mathrm{G}$ Larsson is a scientific founder and holds stock in Pretzel Therapeutics, Inc.

\section{References}

Alam TI, Kanki T, Muta T, Ukaji K, Abe Y, Nakayama H, Takio K, Hamasaki N, Kang D (2003) Human mitochondrial DNA is packaged with tfam. Nucleic Acids Res 31: 1640-1645. doi:10.1093/nar/gkg251

Anderson S, Bankier AT, Barrell BG, de Bruijn MH, Coulson AR, Drouin J, Eperon IC, Nierlich DP, Roe BA, Sanger F, et al (1981) Sequence and organization of the human mitochondrial genome. Nature 290: 457-465. doi:10.1038/290457a0

Bibb MJ, Van Etten RA, Wright CT, Walberg MW, Clayton DA (1981) Sequence and gene organization of mouse mitochondrial DNA. Cell 26: 167-180. doi:10.1016/0092-8674(81)90300-7

Bonekamp NA, Larsson NG (2018) Snapshot: Mitochondrial nucleoid. Cell 172: 388-388.e1. doi:10.1016/j.cell.2017.12.039
Boos F, Krämer L, Groh C, Jung F, Haberkant P, Stein F, Wollweber F, Gackstatter A, Zöller E, van der Laan M, et al (2019) Mitochondrial protein-induced stress triggers a global adaptive transcriptional programme. Nat Cell Biol 21: 442-451. doi:10.1038/s41556-019-0294-5

Brown TA, Tkachuk AN, Shtengel G, Kopek BG, Bogenhagen DF, Hess HF, Clayton DA (2011) Superresolution fluorescence imaging of mitochondrial nucleoids reveals their spatial range, limits, and membrane interaction. Mol Cell Biol 31: 4994-5010. doi:10.1128/ MCB.05694-11

Busch JD, Cipullo M, Atanassov I, Bratic A, Silva Ramos E, Schöndorf T, Li X, Pearce SF, Milenkovic D, Rorbach J, et al (2019) Mitoribo-tag mice provide a tool for in vivo studies of mitoribosome composition. Cell Rep 29: 1728-1738.e9. doi:10.1016/j.celrep.2019.09.080

Cox J, Mann M (2008) Maxquant enables high peptide identification rates, individualized p.P.B.-range mass accuracies and proteome-wide protein quantification. Nat Biotechnol 26: 1367-1372. doi:10.1038/ nbt.1511

Dairaghi DJ, Shadel GS, Clayton DA (1995) Addition of a 29 residue carboxylterminal tail converts a simple hmg box-containing protein into a transcriptional activator. J Mol Biol 249: 11-28. doi:10.1006/ jmbi.1995.9889

Dillon LM, Williams SL, Hida A, Peacock JD, Prolla TA, Lincoln J, Moraes CT (2012) Increased mitochondrial biogenesis in muscle improves aging phenotypes in the mtdna mutator mouse. Hum Mol Genet 21: 2288-2297. doi:10.1093/hmg/dds049

Ekstrand MI, Falkenberg M, Rantanen A, Park CB, Gaspari M, Hultenby K, Rustin P, Gustafsson CM, Larsson NG (2004) Mitochondrial transcription factor a regulates mtdna copy number in mammals. Hum Mol Genet 13: 935-944. doi:10.1093/hmg/ddh109

Falkenberg M, Gaspari M, Rantanen A, Trifunovic A, Larsson NG, Gustafsson CM (2002) Mitochondrial transcription factors b1 and b2 activate transcription of human mtdna. Nat Genet 31: 289-294. doi:10.1038/ ng909

Farge G, Mehmedovic M, Baclayon M, van den Wildenberg SM, Roos WH, Gustafsson CM, Wuite GJ, Falkenberg M (2014) In vitro-reconstituted nucleoids can block mitochondrial DNA replication and transcription. Cell Rep 8: 66-74. doi:10.1016/j.celrep.2014.05.046

Fernandez-Marcos PJ, Auwerx J (2011) Regulation of PGC-1 $\alpha$, a nodal regulator of mitochondrial biogenesis. Am J Clin Nutr 93: 884S-890S. doi:10.3945/ajcn.110.001917

Filograna R, Koolmeister C, Upadhyay M, Pajak A, Clemente P, Wibom R, Simard ML, Wredenberg A, Freyer C, Stewart JB, et al (2019) Modulation of mtdna copy number ameliorates the pathological consequences of a heteroplasmic mtdna mutation in the mouse. Sci Adv 5: eaav9824. doi:10.1126/sciadv.aav9824

Filograna R, Mennuni M, Alsina D, Larsson NG (2021) Mitochondrial DNA copy number in human disease: The more the better?. FEBS Lett 595: 976-1002. doi:10.1002/1873-3468.14021

Fisher RP, Clayton DA (1988) Purification and characterization of human mitochondrial transcription factor 1. Mol Cell Biol 8: 3496-3509. doi:10.1128/mcb.8.8.3496-3509.1988

Fusté JM, Wanrooij S, Jemt E, Granycome CE, Cluett TJ, Shi Y, Atanassova N, Holt IJ, Gustafsson CM, Falkenberg M (2010) Mitochondrial rna polymerase is needed for activation of the origin of light-strand DNA replication. Mol Cell 37: 67-78. doi:10.1016/j.molcel.2009.12.021

Garstka HL, Schmitt WE, Schultz J, Sogl B, Silakowski B, Pérez-Martos A, Montoya J, Wiesner RJ (2003) Import of mitochondrial transcription factor a (tfam) into rat liver mitochondria stimulates transcription of mitochondrial DNA. Nucleic Acids Res 31: 5039-5047. doi:10.1093/nar/ gkg717

Ghazal N, Peoples JN, Mohiuddin TA, Kwong JQ (2021) Mitochondrial functional resilience after tfam ablation in the adult heart. Am J Physiol Cell Physiol 320: C929-C942. doi:10.1152/ajpcell.00508.2020 
Gustafsson CM, Falkenberg M, Larsson NG (2016) Maintenance and expression of mammalian mitochondrial DNA. Annu Rev Biochem 85: 133-160. doi:10.1146/annurev-biochem-060815-014402

Han M, Grunstein M (1988) Nucleosome loss activates yeast downstream promoters in vivo. Cell 55: 1137-1145. doi:10.1016/0092-8674(88)90258-9

Hayashi Y, Yoshida M, Yamato M, Ide T, Wu Z, Ochi-Shindou M, Kanki T, Kang D, Sunagawa K, Tsutsui H, et al (2008) Reverse of age-dependent memory impairment and mitochondrial DNA damage in microglia by an overexpression of human mitochondrial transcription factor a in mice. J Neurosci 28: 8624-8634. doi:10.1523/JNEUROSCI.1957-08.2008

Hillen HS, Morozov YI, Sarfallah A, Temiakov D, Cramer P (2017) Structural basis of mitochondrial transcription initiation. Cell 171: 1072-1081.e10. doi:10.1016/j.cell.2017.10.036

Hokari M, Kuroda S, Kinugawa S, Ide T, Tsutsui H, Iwasaki Y (2010) Overexpression of mitochondrial transcription factor a (tfam) ameliorates delayed neuronal death due to transient forebrain ischemia in mice. Neuropathology 30: 401-407. doi:10.1111/j.14401789.2009.01086.x

Holt IJ, Harding AE, Morgan-Hughes JA (1988) Deletions of muscle mitochondrial DNA in patients with mitochondrial myopathies. Nature 331: 717-719. doi:10.1038/331717a0

Ikeuchi M, Matsusaka H, Kang D, Matsushima S, Ide T, Kubota T, Fujiwara T, Hamasaki N, Takeshita A, Sunagawa K, et al (2005) Overexpression of mitochondrial transcription factor a ameliorates mitochondrial deficiencies and cardiac failure after myocardial infarction. Circulation 112: 683-690. doi:10.1161/CIRCULATIONAHA.104.524835

Jiang M, Kauppila TES, Motori E, Li X, Atanassov I, Folz-Donahue K, Bonekamp NA, Albarran-Gutierrez S, Stewart JB, Larsson NG (2017) Increased total mtdna copy number cures male infertility despite unaltered mtdna mutation load. Cell Metab 26: 429-436.e4. doi:10.1016/ j.cmet.2017.07.003

Jiang M, Xie X, Zhu X, Jiang S, Milenkovic D, Misic J, Shi Y, Tandukar N, Li X, Atanassov I, et al (2021) The mitochondrial single-stranded DNA binding protein is essential for initiation of mtdna replication. Sci Adv 7: eabf8631. doi:10.1126/sciadv.abf8631

Kanki T, Ohgaki K, Gaspari M, Gustafsson CM, Fukuoh A, Sasaki N, Hamasaki N, Kang D (2004) Architectural role of mitochondrial transcription factor a in maintenance of human mitochondrial DNA. Mol Cell Biol 24: 9823-9834. doi:10.1128/MCB.24.22.9823-9834.2004

Kaufman BA, Durisic N, Mativetsky JM, Costantino S, Hancock MA, Grutter P, Shoubridge EA (2007) The mitochondrial transcription factor tfam coordinates the assembly of multiple DNA molecules into nucleoid-like structures. Mol Biol Cell 18: 3225-3236. doi:10.1091/mbc.e07-05-0404

Kauppila JHK, Bonekamp NA, Mourier A, Isokallio MA, Just A, Kauppila TES, Stewart JB, Larsson NG (2018) Base-excision repair deficiency alone or combined with increased oxidative stress does not increase mtdna point mutations in mice. Nucleic Acids Res 46: 6642-6669. doi:10.1093/ nar/gky456

Kinoshita E, Kinoshita-Kikuta E (2011) Improved phos-tag sds-page under neutral ph conditions for advanced protein phosphorylation profiling. Proteomics 11: 319-323. doi:10.1002/pmic.201000472

Kornberg RD, Lorch Y (2020) Primary role of the nucleosome. Mol Cell 79: 371-375. doi:10.1016/j.molcel.2020.07.020

Kucej M, Kucejova B, Subramanian R, Chen XJ, Butow RA (2008) Mitochondrial nucleoids undergo remodeling in response to metabolic cues. J Cell Sci 121: 1861-1868. doi:10.1242/jcs.028605

Kukat C, Davies KM, Wurm CA, Spåhr H, Bonekamp NA, Kühl I, Joos F, Polosa PL, Park CB, Posse V, et al (2015) Cross-strand binding of tfam to a single mtdna molecule forms the mitochondrial nucleoid. Proc Natl Acad Sci U S A 112: 11288-11293. doi:10.1073/pnas.1512131112

Kukat C, Wurm CA, Spåhr H, Falkenberg M, Larsson NG, Jakobs S (2011) Superresolution microscopy reveals that mammalian mitochondrial nucleoids have a uniform size and frequently contain a single copy of mtdna. Proc Natl Acad Sci U S A 108: 13534-13539. doi:10.1073/ pnas. 1109263108

Larsson NG, Wang J, Wilhelmsson H, Oldfors A, Rustin P, Lewandoski M, Barsh GS, Clayton DA (1998) Mitochondrial transcription factor a is necessary for mtdna maintenance and embryogenesis in mice. Nat Genet 18: 231-236. doi:10.1038/ng0398-231

Lorch Y, LaPointe JW, Kornberg RD (1987) Nucleosomes inhibit the initiation of transcription but allow chain elongation with the displacement of histones. Cell 49: 203-210. doi:10.1016/0092-8674(87)90561-7

Lu B, Lee J, Nie X, Li M, Morozov YI, Venkatesh S, Bogenhagen DF, Temiakov D, Suzuki CK (2013) Phosphorylation of human tfam in mitochondria impairs DNA binding and promotes degradation by the aaa+ lon protease. Mol Cell 49: 121-132. doi:10.1016/j.molcel.2012.10.023

Maniura-Weber K, Goffart S, Garstka HL, Montoya J, Wiesner RJ (2004) Transient overexpression of mitochondrial transcription factor a (tfam) is sufficient to stimulate mitochondrial DNA transcription, but not sufficient to increase mtdna copy number in cultured cells. Nucleic Acids Res 32: 6015-6027. doi:10.1093/nar/gkh921

Matic S, Jiang M, Nicholls TJ, Uhler JP, Dirksen-Schwanenland C, Polosa PL, Simard ML, Li X, Atanassov I, Rackham O, et al (2018) Mice lacking the mitochondrial exonuclease mgme1 accumulate mtdna deletions without developing progeria. Nat Commun 9: 1202. doi:10.1038/s41467018-03552-x

Matsushima Y, Goto Y, Kaguni LS (2010) Mitochondrial lon protease regulates mitochondrial DNA copy number and transcription by selective degradation of mitochondrial transcription factor a (tfam). Proc Natl Acad Sci U S A 107: 18410-18415. doi:10.1073/pnas.1008924107

Matsushima Y, Matsumura K, Ishii S, Inagaki H, Suzuki T, Matsuda Y, Beck K, Kitagawa Y (2003) Functional domains of chicken mitochondrial transcription factor a for the maintenance of mitochondrial DNA copy number in lymphoma cell line dt40. J Biol Chem 278: 31149-31158. doi:10.1074/jbc.M303842200

Morimoto N, Miyazaki K, Kurata T, Ikeda Y, Matsuura T, Kang D, Ide T, Abe K (2012) Effect of mitochondrial transcription factor a overexpression on motor neurons in amyotrophic lateral sclerosis model mice. J Neurosci Res 90: 1200-1208. doi:10.1002/jnr.23000

Ngo HB, Kaiser JT, Chan DC (2011) The mitochondrial transcription and packaging factor tfam imposes a u-turn on mitochondrial DNA. Nat Struct Mol Biol 18: 1290-1296. doi:10.1038/nsmb.2159

Nicholls TJ, Nadalutti CA, Motori E, Sommerville EW, Gorman GS, Basu S, Hoberg E, Turnbull DM, Chinnery PF, Larsson NG, et al (2018) Topoisomerase $3 \alpha$ is required for decatenation and segregation of human mtDNA. Mol Cell 69: 9-23.e6. doi:10.1016/j.molcel.2017.11.033

Nishiyama S, Shitara H, Nakada K, Ono T, Sato A, Suzuki H, Ogawa T, Masaki H, Hayashi J, Yonekawa H (2010) Over-expression of tfam improves the mitochondrial disease phenotypes in a mouse model system. Biochem Biophys Res Commun 401: 26-31. doi:10.1016/ j.bbrc.2010.08.143

Niwa H, Yamamura K, Miyazaki J (1991) Efficient selection for high-expression transfectants with a novel eukaryotic vector. Gene 108: 193-199. doi:10.1016/0378-1119(91)90434-d

Okabe M, Ikawa M, Kominami K, Nakanishi T, Nishimune Y (1997) ‘Green mice’ as a source of ubiquitous green cells. FEBS Lett 407: 313-319. doi:10.1016/s0014-5793(97)00313-x

Parisi MA, Clayton DA (1991) Similarity of human mitochondrial transcription factor 1 to high mobility group proteins. Science 252: 965-969. doi:10.1126/science.2035027

Perez-Riverol Y, Csordas A, Bai J, Bernal-Llinares M, Hewapathirana S, Kundu DJ, Inuganti A, Griss J, Mayer G, Eisenacher M, et al (2019) The pride database and related tools and resources in 2019: Improving support for quantification data. Nucleic Acids Res 47: D442-D450. doi:10.1093/ nar/gky1106 
Puigserver P, Wu Z, Park CW, Graves R, Wright M, Spiegelman BM (1998) A coldinducible coactivator of nuclear receptors linked to adaptive thermogenesis. Cell 92: 829-839. doi:10.1016/s0092-8674(00)81410-5

Rahman S (2020) Mitochondrial disease in children. J Intern Med 287: 609-633. doi:10.1111/joim.13054

Ramos SE, Motori E, Brüser C, Kühl I, Yeroslaviz A, Ruzzenente B, Kauppila JHK, Busch JD, Hultenby K, Habermann BH, et al (2019) Mitochondrial fusion is required for regulation of mitochondrial DNA replication. PLOS Genet 15: e1008085. doi:10.1371/journal.pgen.1008085

Rappsilber J, Ishihama Y, Mann M (2003) Stop and go extraction tips for matrix-assisted laser desorption/ionization, nanoelectrospray, and Ic/ms sample pretreatment in proteomics. Anal Chem 75: 663-670. doi:10.1021/ac026117i

R Core Team (2018) A Language and Environment for Statistical Computing. Vienna, Austria: R Foundation for Statistical Computing.

Ringel R, Sologub M, Morozov YI, Litonin D, Cramer P, Temiakov D (2011) Structure of human mitochondrial rna polymerase. Nature 478: 269-273. doi:10.1038/nature10435

Ritchie ME, Phipson B, Wu D, Hu Y, Law CW, Shi W, Smyth GK (2015) Limma powers differential expression analyses for rna-sequencing and microarray studies. Nucleic Acids Res 43: e47. doi:10.1093/nar/gkv007

Rubio-Cosials A, Sidow JF, Jiménez-Menéndez N, Fernández-Millán P, Montoya J, Jacobs HT, Coll M, Bernadó P, Solà M (2011) Human mitochondrial transcription factor a induces a $u$-turn structure in the light strand promoter. Nat Struct Mol Biol 18: 1281-1289. doi:10.1038/ nsmb.2160

Russell OM, Gorman GS, Lightowlers RN, Turnbull DM (2020) Mitochondrial diseases: Hope for the future. Cell 181: 168-188. doi:10.1016/ j.cell.2020.02.051

Scarpulla RC (2002) Nuclear activators and coactivators in mammalian mitochondrial biogenesis. Biochim Biophys Acta 1576: 1-14. doi:10.1016/s0167-4781(02)00343-3

Sciacco M, Bonilla E, Schon EA, DiMauro S, Moraes CT (1994) Distribution of wild-type and common deletion forms of mtdna in normal and respiration-deficient muscle fibers from patients with mitochondrial myopathy. Hum Mol Genet 3: 13-19. doi:10.1093/hmg/3.1.13

Shen EL, Bogenhagen DF (2001) Developmentally-regulated packaging of mitochondrial DNA by the hmg-box protein mttfa during xenopus oogenesis. Nucleic Acids Res 29: 2822-2828. doi:10.1093/nar/29.13.2822

Shi Y, Dierckx A, Wanrooij PH, Wanrooij S, Larsson NG, Wilhelmsson LM, Falkenberg M, Gustafsson CM (2012) Mammalian transcription factor a is a core component of the mitochondrial transcription machinery. Proc Natl Acad Sci U S A 109: 16510-16515. doi:10.1073/pnas.1119738109

Stephan T, Roesch A, Riedel D, Jakobs S (2019) Live-cell sted nanoscopy of mitochondrial cristae. Sci Rep 9: 12419. doi:10.1038/s41598-019-488382

Sterky FH, Lee S, Wibom R, Olson L, Larsson NG (2011) Impaired mitochondrial transport and parkin-independent degeneration of respiratory chaindeficient dopamine neurons in vivo. Proc Natl Acad Sci U S A 108: 12937-12942. doi:10.1073/pnas.1103295108
Taylor RW, Turnbull DM (2005) Mitochondrial DNA mutations in human disease. Nat Rev Genet 6: 389-402. doi:10.1038/nrg1606

Thompson K, Collier JJ, Glasgow RIC, Robertson FM, Pyle A, Blakely EL, Alston CL, Oláhová M, McFarland R, Taylor RW (2020) Recent advances in understanding the molecular genetic basis of mitochondrial disease. J Inherit Metab Dis 43: 36-50. doi:10.1002/jimd.12104

Vafai SB, Mootha VK (2012) Mitochondrial disorders as windows into an ancient organelle. Nature 491: 374-383. doi:10.1038/nature11707

Virbasius JV, Scarpulla RC (1994) Activation of the human mitochondrial transcription factor a gene by nuclear respiratory factors: A potential regulatory link between nuclear and mitochondrial gene expression in organelle biogenesis. Proc Natl Acad Sci U S A 91: 1309-1313. doi:10.1073/pnas.91.4.1309

Viscomi C, Bottani E, Civiletto G, Cerutti R, Moggio M, Fagiolari G, Schon EA, Lamperti C, Zeviani M (2011) In vivo correction of COX deficiency by activation of the AMPK/PGC-1 $\alpha$ axis. Cell Metab 14: 80-90. doi:10.1016/ j.cmet.2011.04.011

Wallace DC, Singh G, Lott MT, Hodge JA, Schurr TG, Lezza AM, Elsas LJ, 2nd, Nikoskelainen EK (1988) Mitochondrial DNA mutation associated with leber's hereditary optic neuropathy. Science 242: 1427-1430. doi:10.1126/science.3201231

Wanrooij S, Fusté JM, Farge G, Shi Y, Gustafsson CM, Falkenberg M (2008) Human mitochondrial rna polymerase primes lagging-strand DNA synthesis in vitro. Proc Natl Acad Sci U S A 105: 11122-11127. doi:10.1073/ pnas.0805399105

Whitaker RM, Corum D, Beeson CC, Schnellmann RG (2016) Mitochondrial biogenesis as a pharmacological target: A new approach to acute and chronic diseases. Annu Rev Pharmacol Toxicol 56: 229-249. doi:10.1146/annurev-pharmtox-010715-103155

Wredenberg A, Wibom R, Wilhelmsson H, Graff C, Wiener HH, Burden SJ, Oldfors A, Westerblad H, Larsson NG (2002) Increased mitochondrial mass in mitochondrial myopathy mice. Proc Natl Acad Sci U S A 99: 15066-15071. doi:10.1073/pnas.232591499

Wu Z, Puigserver P, Andersson U, Zhang C, Adelmant G, Mootha V, Troy A, Cinti S, Lowell B, Scarpulla RC, et al (1999) Mechanisms controlling mitochondrial biogenesis and respiration through the thermogenic coactivator pgc-1. Cell 98: 115-124. doi:10.1016/S0092-8674(00)80611-X

Ylikallio E, Tyynismaa H, Tsutsui H, Ide T, Suomalainen A (2010) High mitochondrial DNA copy number has detrimental effects in mice. Hum Mol Genet 19: 2695-2705. doi:10.1093/hmg/ddq163

Zelenaya-Troitskaya O, Newman SM, Okamoto K, Perlman PS, Butow RA (1998) Functions of the high mobility group protein, abf2p, in mitochondrial DNA segregation, recombination and copy number in saccharomyces cerevisiae. Genetics 148: 1763-1776. doi:10.1093/genetics/148.4.1763

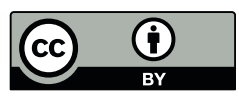

License: This article is available under a Creative Commons License (Attribution 4.0 International, as described at https://creativecommons.org/ licenses/by/4.0/). 